\title{
Estimation of Multivariate Stochastic Volatility Models: A Comparative Monte Carlo Study
}

\author{
M. Hakan Eratalay ${ }^{\circledR}$ \\ European University at St. Petersburg \\ Received: 20.10.2015 Accepted: 30.08.2016 Published: 30.09.2016 \\ doi:10.33818/ier.278044
}

\begin{abstract}
In this paper, we compare the small sample performances of Quasi Maximum Likelihood (QML) and Monte Carlo Likelihood (MCL) methods through Monte Carlo studies for several multivariate stochastic volatility models, among which we consider two new models that account for leverage effects. Our results confirm previous findings within the literature, namely, that the MCL estimator has better finite sample performance compared to the QML estimator. QML estimator's performance is closer to that of MCL estimator when the volatility processes have higher variance or when the correlations are high and/or time varying, but it performs relatively worse when leverage is introduced. Finally, we include an empirical illustration by estimating an MSV model with leverage using a trivariate data from the major European stock markets.
\end{abstract}

Key words: Multivariate Stochastic Volatility, Estimation, Constant Correlations, Time Varying Correlations, Leverage

JEL Classifications: C32, C51, C58

\section{INTRODUCTION}

In financial time series literature, it is already established that the volatilities of asset returns are changing over time. Moreover, they are clustered; i.e. higher (lower) values of volatilities are followed by higher (lower) values, which implies that the volatilities are serially correlated. To capture this kind of dynamic volatility effect, the generalized autoregressive conditional heteroskedasticity (GARCH) models have been proposed by Engle (1982) and Bollerslev (1986). In GARCH models the time varying volatility is modelled as a deterministic function of squared previous day returns and previous day volatilities; therefore, volatilities are observation driven in the GARCH approach. Currently a wide range of GARCH models are available in the literature and are well documented in surveys: see Bollerslev et al. (1992) for univariate and Bauwens et al. (2006), Silvennoinen and Teräsvirta (2009) for multivariate models.

\footnotetext{
${ }^{\circledR}$ M. Hakan Eratalay, Named Professor of Financial Econometrics in the Department of Economics at the European University at St. Petersburg. Professorship position financed by the MDM Bank, (email: heratalay@eu.spb.ru).

I would like to thank the editors and two anonymous referees for their useful comments. I was at the University of Alicante when developing the first draft of this paper. I am grateful to Siem Jan Koopman, Esther Ruiz and M. Angeles Carnero for their helpful comments on the earlier drafts of this paper. I also thank the participants of SAEe 2011, Etsern Meeting 2011, ICEEE 2013 and 33rd Research Conference NES 2013 for their useful comments. Finally research support from MDM Bank (Russia) is gratefully acknowledged. All remaining errors are in my responsibility.
} 
An alternative approach to modelling time varying volatility is to consider it as an unobserved component and let the logarithm of it follow an autoregressive process. Therefore, in this approach, the volatilities are parameter driven. Models of this kind are named as stochastic volatility (SV) models in the literature. The SV approach is attractive because of its similarity to the models used in financial theory to describe the behavior of prices; see Hull and White (1987), Taylor (1986, 1994), and also, Shephard and Andersen (2009). Moreover, it has been shown that SV models describe the behavior of volatilities more accurately compared to GARCH models (see for example Danielsson, 1994; Kim et al., 1998; and Carnero et al., 2004). Given the way the SV models are set up, their statistical properties are easy to derive from the process that the volatilities follow. However, although statistically more attractive than GARCH models, SV models have a disadvantage in terms of estimation, because their exact likelihoods are difficult to evaluate. The following survey papers are available about the univariate and multivariate SV (MSV) models and estimation methods: Broto and Ruiz (2004), Asai et al. (2006), Chib et al. (2009), Ghysels et al. (1996), Yu and Meyer (2006), Maasoumi and McAleer (2006), and Omori and Ishihara (2012).

Several methods have been proposed for estimating SV models. A relatively easy approach is the quasi-maximum likelihood estimation (QML), proposed independently by Nelson (1988) and Harvey et al. (1994). In this approach, the logarithms of squared returns (hereforth referred to as log-squared returns) are modelled as a linear state space form where the transformed innovations are assumed to follow a Gaussian distribution, although, in fact, the true distribution is based on $\ln \chi_{1}^{2}$ (see Sandmann and Koopman, 1998, for the univariate and Asai and McAleer, 2006, for the multivariate case). Ruiz (1994) showed that the QML estimators are consistent and asymptotically normal. However, due to the Gaussianity assumption, QML approach is an estimation based on approximations and therefore, it is inefficient as noted in the simulation studies by several papers as Jacquier et al. (1994), Breidt and Carriquiry (1996) and Sandmann and Koopman (1998). Also, the transformed returns might take very large negative values (or even minus infinity), which is known as the inlier problem ${ }^{1}$.

One way to avoid this inefficiency problem is to evaluate the exact likelihood. This requires high dimensional integration, which could be achieved by evaluating these integrals with simulation methods and then maximizing the resulting likelihood function. This class of simulated likelihood methods include the accelerated importance sampling (AGIS) approach developed in Danielsson and Richard (1993), efficient importance sampling (EIS) approach proposed by Liesenfeld and Richard (2003, 2006), and the Monte Carlo likelihood (MCL) approach proposed by Sandmann and Koopman (1998) and Jungbacker and Koopman (2006). The novelty of the MCL method is that simulations are only needed for a small part of the likelihood function, unlike in the case of other simulated likelihood methods. Hence, it is computationally less intensive ${ }^{2}$. Different from the QML estimation, the MCL method of Sandmann and Koopman (1998) used log-squared transformation of returns taking into account the true distribution of the errors and therefore was modelling the log-squared returns via a linear non-Gaussian state space model. However, as QML method, MCL method of Sandmann and Koopman (1998) also has to adjust for the inlier problem. A review of these importance

\footnotetext{
${ }^{1}$ See Section 2.4.1. for an explanation on how this inlier problem is treated.

${ }^{2}$ Durbin and Koopman (1997) showed that the loglikelihood of the state space models with non-Gaussian errors can be written as a sum of the loglikelihood of the approximating Gaussian model and a correction for the departures from the Gaussianity assumption with respect to the true model. This form of loglikelihood has the advantage that the simulations are only required for the departures of the loglikelihood of the true model from the Gaussian loglikelihood, rather than for the whole loglikelihood itself. See also Asai et al. (2006).
} 
sampling methods could be found again in Asai et al. (2006) ${ }^{3}$. Below is a nonexhaustive comparison of these estimation methods.

The MCL method considered in this paper is the one proposed by Jungbacker and Koopman (2006) that extended the theoretical results of Shephard and Pitt (1997), Durbin and Koopman (1997), and Jungbacker and Koopman (2005). In this method the returns are modelled without the log-squared transformation, hence there is no inlier problem. Jungbacker and Koopman (2006) used this approach to estimate three multivariate stochastic volatility (MSV) models: the stochastic time varying scaling factor model, where the variance matrix of the returns are scaled by the log-volatilities, the Constant Correlation MSV model of Harvey et al. (1994) and the Time Varying Correlation MSV model based on Cholesky decomposition. In the latter set up, the correlation dynamics is driven by the volatilities and a correlation parameter ${ }^{4}$.

\begin{tabular}{|c|c|c|c|}
\hline Estimation method & Pros & Cons & Empirical Examples \\
\hline $\begin{array}{l}\text { QML } \\
\text { H. et al. }(1994)^{\mathrm{a}} \\
\text { R. }(1994)^{\mathrm{b}}\end{array}$ & $\begin{array}{l}\text { Consistent, } \\
\text { asymptotically normal, } \\
\text { implementation is easier }\end{array}$ & $\begin{array}{l}\text { Inefficient, inlier } \\
\text { problem }\end{array}$ & $\begin{array}{l}\text { Harvey and Shephard (1996) } \\
\text { Hwang and Satchell (2000) } \\
\text { Yu (2002), Alizadeh et al. (2002) }\end{array}$ \\
\hline $\begin{array}{l}\text { SML } \\
\text { D. \& R.. }(1993)^{\mathrm{c}} \\
\text { L. \& R. }(2003,2006)^{\mathrm{d}}\end{array}$ & $\begin{array}{l}\text { Consistent, } \\
\text { asymptotically normal, } \\
\text { efficient }\end{array}$ & $\begin{array}{l}\text { Computationally } \\
\text { intensive }\end{array}$ & $\begin{array}{l}\text { Danielsson (1998) } \\
\text { Liesenfeld (1998, 2001) } \\
\text { Liesenfeld and Jung (2000) }\end{array}$ \\
\hline $\begin{array}{l}\text { MCL } \\
\text { S. \& K. }(1998)^{\mathrm{e}} \\
(\text { J. \& K. 2006) }\end{array}$ & $\begin{array}{l}\text { Consistent, } \\
\text { asymptotically normal, } \\
\text { efficient, computationally } \\
\text { less intensive than SML }\end{array}$ & $\begin{array}{l}\text { In MCL of } \\
\text { S. \& K. (1998) } \\
\text { inlier problem }\end{array}$ & $\begin{array}{l}\text { Asai and McAleer }(2005,2006) \\
\text { Brandt and Kang }(2004) \\
\text { Asai and Unite (2008) }\end{array}$ \\
\hline
\end{tabular}

Table 1.1 Nonexhaustive comparison of estimation methods.

Sources: Asai et al. (2006), ${ }^{\mathrm{a}}$ Harvey et al. (1994), ${ }^{\mathrm{b}}$ Ruiz (1994), ${ }^{\mathrm{c}}$ Danielsson and Richard (1993), ${ }^{\mathrm{d}}$ Liesenfeld and Richard (2003, 2006), ${ }^{\mathrm{e}}$ Sandmann and Koopman (1998), ${ }^{\mathrm{f}}$ Jungbacker and Koopman (2006).

When fitting an MSV model to a financial time series, researchers are ultimately interested in estimating the underlying volatilities and correlations ${ }^{5}$. Therefore, when making a comparison of performances between different estimators, one should also consider looking at their relative performances in estimating the in-sample volatilities and correlations. In this respect, we employ several Monte Carlo (MC) experiments where the performances of QML and MCL methods in estimating the parameters, volatilities and correlations are compared. It is already known that MCL methods, being a maximum likelihood method, is asymptotically efficient. However, QML method is based on approximations and, hence, inefficient in parameter estimation. (See Sandmann and Koopman, 1998; Jacquier et al., 1994; among others). The performance of the QML estimator suffers from the fact that the true transformed errors are asymmetrically distributed. On the other hand, the QML method is much easier to implement and is easily adaptable to estimate with higher number of series or higher number of observations. In the literature there is a need for Monte Carlo simulation studies comparing QML and MCL methods, in terms of in-sample volatility and correlation estimations in a multivariate setup and for different parameter sets. In this paper, we contribute to filling this gap in the literature with a number of MC experiments for several models, and we discuss when the QML method performs closer to MCL method and, hence, can be used.

\footnotetext{
${ }^{3}$ Finally, the Monte Carlo Markov Chain (MCMC) methods are receiving much attention since they provide the most efficient estimation tools (see Andersen et al., 1999). For a survey on MCMC methods and MCMC estimation of several MSV models, see Asai et al. (2006), Meyer and Yu (2000), Chib et al. (2009). MCMC method will be outside the scope of this paper.

4 Tsay (2005) adopted a Cholesky decomposition based approach to ensure the positive definiteness of the covariance matrix. The MSV model he proposed is basically the same time varying correlation MSV model as considered in Jungbacker and Koopman (2006) with the correlation parameter following a stochastic autoregressive process.

${ }^{5}$ Throughout this paper, correlations refer to the correlations between returns, if not otherwise stated.
} 
When it is of interest to estimate models with high number of series, the implementation of the QML estimator is easier and more feasible than that of the MCL estimator. Moreover, the explicit analytical derivatives needed for the MCL estimation may be harder to obtain with large cross-sections. One could choose to use numerical derivatives, but the derivatives obtained by numerical approximation for large state vectors could be very time consuming and numerically unstable. Hence, it is interesting to know when we can use the QML estimator instead of MCL estimator, the latter being computationally more costly.

For our MC experiments, we first consider the Constant Correlation MSV model of Harvey et al. (1994). As pointed out by Tsui and Yu (1999), the correlations do not have to be constant for certain assets. For this reason, we also consider the Time Varying Correlation MSV model discussed in Jungbacker and Koopman $(2006)^{6}$. Another stylized fact is the so called leverage effect, which refers to the negative relation between the current returns and future volatilities. Black (1976) and Christie (1982) found that there is a negative relation between the ex-post volatility of the return rates on assets and the current value of the asset. One way to explain this is that decreasing prices of assets (negative returns) imply an increased leverage of the firms, which is believed to increase uncertainty and, hence, volatility. (See Ghysels et al., 1996). Jungbacker and Koopman (2005) proposed a univariate SV model with leverage and discussed how to estimate it via the MCL method. In our paper we discuss a direct multivariate generalization of this model and refer to it as MSV with diagonal leverage, where the correlations between the standardized innovations of returns and volatilities are diagonal ${ }^{7}$. Furthermore, we discuss the MSV model with non-diagonal leverage, where the correlations between the standardized innovations of returns and volatilities are non-diagonal; i.e. the standardized innovations of the volatility of series $i$ is correlated with the standardized innovations of the returns of series $j$. We also provide the necessary transformations to estimate these two MSV with leverage models via MCL method, which are derived based on the univariate estimation in Jungbacker and Koopman (2005). A similar model has been proposed by Asai and McAleer (2006) that considers a diagonal leverage between the non-standardized innovations ${ }^{8}$. They estimated this model via the MCL method of Sandmann and Koopman (1998). The two MSV models with leverage discussed in this paper imply a nondiagonal leverage matrix in the context of the model of Asai and McAleer (2006, see Appendix 6.2). Moreover, the leverage coefficients in their model are proportional to the standard deviations of the volatility equation errors, which is a restriction that we do not impose in our models.

The results obtained in this paper confirm that QML estimator has worse finite sample performance than MCL estimator. However, under the constant correlations assumption, when the true value of the underlying correlation is high and/or when the variances of the SV processes are high, the QML estimator is performing closer to the MCL estimator. When we allow the correlations to vary over time, the performance of the QML estimator approaches to that of the MCL estimator, even with lower correlations. When leverage is allowed in the model, the performance of the QML estimator is worse in estimating the underlying correlations, compared to its performance in the model without leverage ${ }^{9}$. Based on our results, we conclude that the QML estimator could be used when the series are expected to have high correlations

\footnotetext{
${ }^{6}$ For time varying correlation MSV models also see Yu and Meyer (2006), Tsay (2005) among others.

${ }^{7}$ By standardized innovations, we mean innovations standardized by the variance matrices.

${ }^{8}$ There are other MSV models with leverage proposed by these authors. See Asai and McAleer (2005, 2009, 2011, 2015).

${ }^{9}$ Given that the true distribution of the log-squared errors is asymmetric and leverage makes this asymmetry worse, the decrease in the performance of QML estimator is expected. Thanks to an anonymous referee for pointing this out.
} 
(whether constant or time varying) and when the variances of the SV processes are high. Particularly in the case of MSV models with leverage we do not recommend the use of the QML estimator ${ }^{10}$.

The paper is organized as follows: in Section 2.1-2.3 we briefly discuss stochastic volatility models, and later in Section 2.4, we provide information on how these models can be estimated via QML and MCL methods. In Section 3, we explain the set up of our Monte Carlo experiments and discuss the results. In Section 4, we estimate a trivariate MSV model with leverage for the returns on three major European stock markets. Finally, in Section 5, we discuss further topics for research and conclude.

\section{MULTIVARIATE STOCHASTIC VOLATILITY (MSV) MODELS}

\subsection{The Basic Model}

The univariate SV model was proposed by, among others, Taylor (1982, 1986). Harvey et al. (1994) extended this univariate SV model to a multivariate context, proposing the first multivariate SV (MSV) model. If we let $y_{t}=\left(y_{1 t}, y_{2 t}, \ldots, y_{k t}\right)^{\prime}$ be a $k \times 1$ vector of observations at time $t$ and $h_{t}=\left(h_{1 t}, h_{2 t}, \ldots, h_{k t}\right)^{\prime}$ be the corresponding log-volatilities, then this model is defined as:

$$
y_{t}=H_{t}^{1 / 2} \varepsilon_{t}
$$

where $H_{t}=\operatorname{diag}\left\{e^{h_{1 t}}, e^{h_{2 t}}, \mathrm{~K}, e^{h_{k t}}\right\}=\operatorname{diag}\left\{e^{h_{t}}\right\}$

$$
\begin{gathered}
h_{t+1}=\Gamma+\Phi h_{t}+\eta_{t} \\
h_{1} \sim N\left(\left(I_{k}-\Phi\right)^{-1} \Gamma, \Sigma_{0}\right) \\
\left(\begin{array}{l}
\varepsilon_{t} \\
\eta_{t}
\end{array}\right) \sim N\left(0_{2 k},\left[\begin{array}{ll}
P_{\varepsilon} & 0_{k} \\
0_{k} & Q_{\eta}
\end{array}\right]\right)
\end{gathered}
$$

where $\Gamma$ is a $k \times 1$ vector of, and $\Phi$ is a $k \times k$ matrix of parameters. $I_{k}$ denotes a $k \times k$ identity matrix and $0_{k}$ denotes a $k \times k$ matrix of zeros. The covariance matrices $P_{\varepsilon}$ and $Q_{\eta}$ are of the corresponding errors $\varepsilon_{t}$ and $\eta_{t}$. The diagonal elements of $P_{\varepsilon}$ are restricted to be equal to one for identification purposes; therefore $P_{\varepsilon}$ is a correlation matrix. For simplicity, we do not consider volatility spillovers; i.e. $\Phi$ is a diagonal matrix. However, the volatilities $h_{t}$ are still dependent on each other via $Q_{\eta}$ matrix. Finally, the $(i, j)$ element of $\Sigma_{0}$ is the $(i, j)$ element of $Q_{\eta}$ divided by $\left(1-\Phi_{i i} \Phi_{j j}\right) .{ }^{11}$ By construction, this model assumes constant correlation; therefore, following Yu and Meyer (2006), we will refer to this model as the Constant Correlation MSV (CCMSV) model. In our analysis, we focus on the following parameters, in order: $\Psi=\left(\operatorname{vecl}\left(P_{\varepsilon}\right)^{\prime}, \Gamma^{\prime}, \operatorname{diag}(\Phi)^{\prime}, \operatorname{vech}\left(Q_{\eta}\right)^{\prime}\right)^{\prime}{ }^{12}$ In this model there are $k^{2}+2 k$ parameters to estimate.

\subsection{Time Varying Correlation MSV}

\footnotetext{
${ }^{10}$ To know in advance whether data at hand has leverage effects, one could estimate an MGARCH model (for example a multivariate EGARCH model) with leverage/asymmetric effects and analyze the results.

11 That is, $\Sigma_{0}$ satisfies the stationarity condition: $\Sigma_{0}=\Phi \Sigma_{0} \Phi+Q_{\eta}$. Therefore the elements of $\Sigma_{0}$ can be obtained by: $\operatorname{vec}\left(\Sigma_{0}\right)=\left(I_{k^{2}}-\Phi \otimes \Phi\right)^{-1} \operatorname{vec}\left(Q_{\eta}\right)$, where vec is the operator that stacks the columns of a matrix and $\otimes$ is a Kronecker product.

${ }^{12}$ The operator vec stacks all columns of a matrix, while vech stacks the columns of the lower triangular part of a matrix and vecl stacks the columns of the strict lower triangular (exluding the leading diagonal from the lower triangular matrix) part of a matrix.
} 
The Time Varying Correlation MSV model considered in our paper is the one mentioned in Jungbacker and Koopman (2006) ${ }^{13}$. We will refer to this model as TVCMSV. Following the notation above, the observation equation (2.1) is modified as:

$$
\begin{aligned}
& y_{t}=D H_{t}^{1 / 2} \varepsilon_{t} \\
& \varepsilon_{t} \sim N\left(0, I_{k}\right)
\end{aligned}
$$

where $D$ is a lower unity triangular matrix. The idea is to decompose the conditional variance of $y_{t}, \operatorname{Var}\left(y_{t} \mid h_{t}\right)=V_{t}=D H_{t} D^{\prime}$, and therefore having a stochastic dynamics behind the variances and correlations implied by $V_{t}$. If we would call $g_{i i, t}=\exp \left(h_{i, t}\right)$ and $D=\left\{q_{i j} \neq 0\right.$ when $i>j$, 0 otherwise $\}$, then the implied correlations by the model are given by:

$$
\begin{gathered}
\sigma_{i i, t}=\sum_{s=1}^{i} q_{i s}^{2} g_{s s, t}, i=1,2, \mathrm{~K}, k \\
\sigma_{i j, t}=\sum_{s=1}^{j} q_{i s} q_{j s} g_{s s, t}, i>j, i=2,3, \mathrm{~K}, k \\
p_{i j, t}=\frac{\sigma_{i j, t}}{\sqrt{\sigma_{i i, t} \sigma_{j j, t}}}=\frac{\sum_{s=1}^{j} q_{i s} q_{j s} g_{s s, t}}{\sqrt{\sum_{s=1}^{i} q_{i s}^{2} g_{s s, t} \sum_{s=1}^{j} q_{j s}^{2} g_{s s, t}}}
\end{gathered}
$$

This model is also a special case of factor MSV models proposed by Shephard (1996) and further studied in Aguilar and West (2000) and Chib et al. (2006) with the number of factors being equal to the number of series. A shortcoming of this model is that the driving forces underlying the volatility and correlation dynamics are the same; $g_{i i, t}$ and $q_{i j}$. The model parameters are $\Psi=\left(\operatorname{vec} l(D)^{\prime}, \Gamma^{\prime}, \operatorname{diag}(\Phi)^{\prime}, \operatorname{vech}\left(Q_{\eta}\right)^{\prime}\right)^{\prime}$. The number of parameters to be estimated in this model is also given by $k^{2}+2 k$.

Tsay (2005) lets the correlation parameters to be dynamic in the sense that the unity lower triangular matrix $D$ becomes $D_{t}=\left\{q_{i j t} \neq 0\right.$ when $i>j, 0$ otherwise $\}$, where $q_{i j t}$ follows a Gaussian $\mathrm{AR}(1)$ process. Then the equation (2.4) becomes:

$$
y_{t}=D_{t} H_{t}^{1 / 2} \varepsilon_{t}
$$

where the $[k(k-1) / 2] \times 1$ vector $q_{t}$ evolves with the equation:

such that:

$$
\begin{aligned}
& q_{t+1}=\beta+\Psi q_{t}+v_{t} \\
& q_{1} \sim N\left(\left(I_{k}-\Psi\right)^{-1} \beta, \Lambda_{0}\right)
\end{aligned}
$$

$$
\left(\begin{array}{l}
\varepsilon_{t} \\
\eta_{t} \\
v_{t}
\end{array}\right) \sim N\left(0_{3 k},\left[\begin{array}{ccc}
I_{k} & 0_{k} & 0_{k} \\
0_{k} & Q_{\eta} & 0_{k} \\
0_{k} & 0_{k} & \Lambda_{v}
\end{array}\right]\right)
$$

where $\Lambda_{0}$ is defined similar to $\Sigma_{0}$. We can put this model to a state space form as follows: let $\alpha_{t}=\left(h_{t}^{\prime}, q_{t}^{\prime},\right)^{\prime}, \omega_{t}=\left(\left(\eta_{t}\right)^{\prime},\left(v_{t}\right)^{\prime}\right)^{\prime}, \bar{\Gamma}=\left(\Gamma^{\prime}, \beta^{\prime}\right)^{\prime}$ such that:

$$
\alpha_{t+1}=\bar{\Gamma}+\left(\begin{array}{cc}
\Phi & 0_{k} \\
0_{k} & \Psi_{\eta}
\end{array}\right) \alpha_{t}+\omega_{t}
$$

where

\footnotetext{
${ }^{13}$ We chose this model, because its estimation is very similar to that of CCMSV model (see Section 2.4.), and also because it is a simpler version of the model proposed in Tsay (2005) based on Choleski decomposition of the correlation matrix.
} 


$$
\begin{aligned}
& \omega_{t} \sim N\left(0_{2 k},\left[\begin{array}{cc}
Q_{\eta} & 0_{k} \\
0_{k} & \Lambda_{v}
\end{array}\right]\right) \\
& \alpha_{1} \sim N\left(\left(\left(\begin{array}{l}
\left(I_{k}-\Phi\right)^{-1} \Gamma \\
\left(I_{k}-\Psi\right)^{-1} \beta
\end{array}\right),\left[\begin{array}{cc}
\Sigma_{0} & 0_{k} \\
0_{k} & \Lambda_{0}
\end{array}\right]\right)\right.
\end{aligned}
$$

The question of how to estimate the TVCMSV model defined via (2.5) and (2.6) via QML and MCL methods is left for future research. The model parameters are $\Psi=\left(\beta^{\prime}, \operatorname{diag}(\Psi)^{\prime}, \Gamma^{\prime}, \operatorname{diag}(\Phi)^{\prime}, \operatorname{vech}\left(Q_{\eta}\right)^{\prime}\right)^{\prime}$, and the number of parameters to estimate in this model is $k^{2}+5 k$. In our Monte Carlo experiments we only consider the TVCMSV model of Jungbacker and Koopman (2006).

\subsection{MSV with Leverage Effect}

The first MSV model with diagonal leverage we discuss here is a direct generalization of the univariate model considered in Jungbacker and Koopman (2005). Changing the definition of the errors slightly, we could rewrite the equations (2.1), (2.2) and (2.3) of CCMSV model as follows:

$$
\begin{gathered}
y_{t}=H_{t}^{1 / 2} P_{\varepsilon}^{*} \varepsilon_{t} \\
h_{t+1}=\Gamma+\Phi h_{t}+Q_{\eta}^{*} \eta_{t}
\end{gathered}
$$

with the following modification made to the distribution of the errors:

$$
\left(\begin{array}{l}
\varepsilon_{t} \\
\eta_{t}
\end{array}\right) \sim N\left(0_{2 k},\left[\begin{array}{cc}
I_{k} & L \\
L & I_{k}
\end{array}\right]\right)
$$

where $L=\left\{\lambda_{i i}, i=1, \ldots, k: \lambda_{i i} \in[-1,1]\right\}$ is assumed to be a diagonal matrix. Therefore by construction, the MSV with diagonal leverage model defined by equations (2.7) and (2.8) implies constant correlation. A transformation similar to the one in Jungbacker and Koopman (2005) could be then adapted to write this model in a state space form:

$$
\begin{gathered}
y_{t}=H_{t}^{1 / 2} P_{\varepsilon}^{*}\left\{\varepsilon_{t}^{*}+S \eta_{2 t}\right\} \\
h_{t+1}=\Gamma+\Phi h_{t}+Q_{\eta}^{*}\left\{\eta_{1 t}+\eta_{2 t}\right\} \\
\left(\begin{array}{c}
\varepsilon_{t}^{*} \\
\eta_{1 t} \\
\eta_{2 t}
\end{array}\right) \sim N\left(0_{3 k},\left[\begin{array}{ccc}
I_{k}-|L| & 0_{k} & 0_{k} \\
0_{k} & I_{k}-|L| & 0_{k} \\
0_{k} & 0_{k} & |L|
\end{array}\right]\right)
\end{gathered}
$$

where $S$ matrix is a diagonal matrix of the signs of each element of $L$, while $|L|$ is the absolute value of (the elements of) $L$ matrix. (Therefore $S|L|=L$ ). $P_{\varepsilon}^{*}$ and $Q_{\eta}^{*}$ are obtained via Cholesky defactorization of $P_{\varepsilon}$ and $Q_{\eta}$, respectively. The errors are all mutually and serially independent. It can be shown that the transformed model in equation (2.9) is consistent with the MSV model with leverage defined by equations (2.7) and (2.8).

Defining the state and signal vectors as $\alpha_{t}=\left(h_{t}^{\prime},\left(Q_{\eta}^{*} \eta_{2, t}\right)^{\prime}\right)^{\prime}, \eta_{t}=\left(\left(Q_{\eta}^{*} \eta_{1, t}\right)^{\prime},\left(Q_{\eta}^{*} \eta_{2, t+1}\right)^{\prime}\right)^{\prime}$ and $\bar{\Gamma}=\left(\Gamma^{\prime}, 0_{k}\right)^{\prime}$ we have the transformed model ready for MCL estimation:

$$
\begin{aligned}
y_{t} & =H_{t}^{1 / 2} P_{\varepsilon}^{*}\left\{\varepsilon_{t}^{*}+S \eta_{2 t}\right\} \\
\alpha_{t+1} & =\bar{\Gamma}+\left(\begin{array}{cc}
\Phi & I_{k} \\
0_{k} & 0_{k}
\end{array}\right) \alpha_{t}+\eta_{t}
\end{aligned}
$$

where 


$$
\begin{gathered}
\eta_{t} \sim N\left(0_{2 k},\left[\begin{array}{cc}
Q_{\eta}^{*}\left(I_{k}-|L|\right)\left(Q_{\eta}^{*}\right)^{\prime} & 0_{k} \\
0_{k} & Q_{\eta}^{*}|L|\left(Q_{\eta}^{*}\right)^{\prime}
\end{array}\right]\right) \\
\alpha_{1} \sim N\left(\left(\begin{array}{c}
\left(I_{k}-\Phi\right)^{-1} \bar{\Gamma} \\
0_{k}
\end{array}\right), \Omega_{0}\right) \\
\operatorname{vec}\left(\Omega_{0}\right)=\left[I_{4 k^{2}}-\left(\begin{array}{cc}
\Phi & I_{k} \\
0_{k} & 0_{k}
\end{array}\right) \otimes\left(\begin{array}{cc}
\Phi & I_{k} \\
0_{k} & 0_{k}
\end{array}\right)\right]^{-1} \operatorname{vec}\left[\begin{array}{cc}
Q_{\eta}^{*}\left(I_{k}-|L|\right)\left(Q_{\eta}^{*}\right)^{\prime} & 0_{k} \\
0_{k} & Q_{\eta}^{*}|L|\left(Q_{\eta}^{*}\right)^{\prime}
\end{array}\right]
\end{gathered}
$$

where $\bar{\Gamma}$ is defined as above. The parameter vector in this case is $\Psi=\left(\operatorname{vecl}\left(P_{\varepsilon}\right), \Gamma^{\prime}, \operatorname{diag}(\Phi)^{\prime}, \operatorname{vech}\left(Q_{\eta}\right)^{\prime} \text {, vec }(L)^{\prime}\right)^{\prime}$, which has $k^{2}+3 k+k(k-1) / 2$ parameters to estimate.

In the model of Asai and McAleer (2006), the leverage matrix between the nonstandardized errors of return and volatility equations is given by $\bar{L}=\operatorname{diag}\left(\lambda_{1} \sigma_{11}^{1 / 2}, \lambda_{2} \sigma_{22}^{1 / 2}, \ldots, \lambda_{k} \sigma_{k k}^{1 / 2}\right)$ and $Q_{\eta}=\sigma_{\eta, i j}$.If we would calculate the leverage matrix in the same manner for our models we would obtain $\bar{L}=Q_{\eta}^{*} L P_{\varepsilon}^{* \prime}$, which is not a diagonal matrix; i.e. in the models discussed in this section $Q_{\eta}^{*} L P_{\varepsilon}^{* \prime}$, is not diagonal whether $L$ is diagonal or not. Therefore, the two models discussed here offer a richer structure compared to the one of Asai and McAleer (2006). See Appendix 6.2 for the model of Asai and McAleer (2006) and also for further discussion.

Asai and McAleer (2006) estimate their model with the MCL method of Sandmann and Koopman (1998). In Section 4, we estimate the MSV with leverage model of Asai and McAleer (2006) through the MCL method explained in their paper. On the other hand, the estimation of these MSV with leverage models via QML could be done by adopting the transformations in Asai and McAleer (2006) and is discussed in Section 2.4.1. We assume throughout the paper for simplicity that whenever the true $L$ matrix is non-diagonal, it is symmetric. In reality, this is not necessarily the case. Moreover, the symmetricity assumption is not needed for QML estimation but is required for MCL estimation, along with the assumption that $L$ is positive or negative semi-definite.

\subsection{Estimating the MSV Models}

From the models we discussed above, we will consider the CCMSV, TVCMSV and MSV models with leverage. We estimate all these models by both the QML approach of Harvey et al. (1994) and the MCL approach of Jungbacker and Koopman (2006). These estimation methods are briefly explained below ${ }^{14}$.

\subsubsection{Quasi-maximum Likelihood (QML) Estimation}

In this estimation method, the multivariate return vector $y_{t}$ is put through a log-squared transformation in order to obtain a state space formation of the model. For the CCMSV model, the observation equation and the state equation are given as:

\footnotetext{
${ }^{14}$ Originally the CCMSV model proposed in Harvey et al. (1994) was estimated by Quasi-maximum Likelihood approach while Jungbacker and Koopman (2006) estimated it by their Monte Carlo Likelihood approach. The TVCMSV model with deterministic correlation parameter in Jungbacker and Koopman (2006) was estimated via their MCL approach. The univariate MSV model with leverage in Jungbacker and Koopman (2005) was estimated using MCL method of Jungbacker and Koopman (2006) while Asai and McAleer (2006) estimated their model with the MCL approach of Sandman and Koopman (1998).
} 


$$
\begin{aligned}
& \log \left(y_{t}^{2}\right)=h_{t}+\log \left(\varepsilon_{t}^{2}\right)=-1.2703 t+h_{t}+\xi_{t} \\
& h_{t+1}=\Gamma+\Phi h_{t}+\eta_{t}
\end{aligned}
$$

where $l$ is a vector of ones, the mean of $\log \left(\varepsilon_{i t}^{2}\right)$ is -1.2703 , and its variance is $\pi^{2} / 2$. In fact, the distribution of $\log \left(\varepsilon_{i t}^{2}\right)$ is based on a $\ln \left(\chi_{1}^{2}\right)$ distribution. (See for Sandmann and Koopman, 1998, for the univariate and Asai and McAleer, 2006, for the multivariate model). We can replace $\log \left(\varepsilon_{t}^{2}\right)+1.2703 l$ with $\xi_{t}$, whose mean is therefore a vector of zeros and it's covariance matrix is given by $P_{\xi}$, which is defined below. The QML method approximates the distribution of $\xi_{t}$ with $N\left(0, P_{\xi}\right)$. The QML estimation procedure is relatively easy to implement: Kalman filter is applied to the log-squared returns and afterwards, the one-step ahead prediction errors, and their variances are used to obtain the likelihood function. However, this estimation only yields minimum mean square linear estimators, because Kalman filter is a linear filter ${ }^{15}$. Taking into account the non-Gaussian distribution of $\xi_{t}$, the asymptotic standard errors can be obtained following Dunsmuir (1979). Harvey (1989) notes that these asymptotic standard errors can not be used for testing if the parameters in the matrix $Q_{\eta}$ are significantly different from zero. On the other hand usual quasi-maximum likelihood theory applies, and the Bollerslev-Wooldridge (1992) robust standard errors can be used. To estimate the in-sample estimates of volatilities and correlations, a Kalman smoothing algorithm is employed.

In Harvey et al. (1994) the $i j$-th element of the covariance matrix $P_{\xi}$ is given by $\left(\pi^{2} / 2\right) p_{i j}^{*}$, where $p_{i i}^{*}=1$ and:

$$
p_{i j}^{*}=\frac{2}{\pi^{2}} \sum_{s=1}^{\infty} \frac{(s-1) !}{(1 / 2)_{s} s} p_{i j}^{2 s}
$$

where $(x)_{s}=x(x+1) \ldots(x+s-1)$. In our implementation, we first maximize with respect to the variable $p_{i j}^{2}$, and then after the maximization we obtain $\left|p_{i j}^{2}\right|$. The sign of $p_{i j}^{2}$ can be recovered from the sign of the product of the corresponding pair of observations, i.e. $y_{i} y_{j}$. If more than half of the multiplications $y_{i} y_{j}$ are positive, then the sign of $p_{i j}$ is positive.

Although, the QML method provides consistent estimators, because of the Gaussian approximation, it is likely to have poor sample properties. Jacquier et al. (1994), Breidt and Carriquiry (1996), and Sandmann and Koopman (1998) are some of the papers that document the inefficiency of the QML estimation.

One problem with the QML estimation is the existence of inliers, i.e. due to missing data or simply by chance some returns will be zero or very close to zero. Therefore a log-squared transformation of this return will explode. To take care of this, several methods are used in the literature. Kim et al. (1998) considered a transformation such as $\log \left(y_{t}^{2}+\mathrm{c}\right)$, where $c=0.001$, while Fuller (1996) assumed a data driven transformation. We follow here the transformation discussed in Sandmann and Koopman (1998), where the values of $\log \left(y_{t}^{2}\right)$, which are less than -20 , are set equal to -20 .

Ruiz (1994) and Harvey et al. (1994) suggest that the intercept of the SV process could be obtained directly from the observations via a moment estimator, and the loglikelihood is optimized for the rest of the parameters. This could prove useful when the cross section is large. In fact, this approach could also be used for the MCL estimation when the errors are assumed to be Gaussian as in QML estimation. However, in this paper we preferred to estimate all parameters by maximizing the loglikelihood.

\footnotetext{
${ }^{15}$ How to improve the performance of QML estimators in a multivariate setting using a nonlinear filter is an interesting topic for future research. Watanabe (1999) used a nonlinear filtering to improve the performance of QML estimators in a univariate setting.
} 
The estimation of TVCMSV model via QML method is very similar to the estimation of the CCMSV model. The only difference is that in the estimation, the log-squared transformation should be applied to $D^{-1} y_{t}$ and the resulting loglikelihood function contains an additional term: $T \log (\operatorname{det}(D))$. Given that in the TVCMSV set up in our paper the $D$ matrix is lower unity triangular, its determinant is one, and therefore, this additional term is equal to zero ${ }^{16}$.

For estimating the MSV model with (diagonal or nondiagonal) leverage via QML method, the log-squared transformation as discussed in Asai and McAleer (2006) can be applied to the model:

where

$$
\begin{aligned}
& \log \left(y_{t}^{2}\right)=h_{t}+\log \left(\varepsilon_{t}^{2}\right)=h_{t}+\xi_{t} \\
& h_{t+1}=\Gamma+\Gamma_{t}^{*}+\Phi h_{t}+\eta_{t}^{*}
\end{aligned}
$$

$$
\begin{aligned}
& \eta_{t}^{*} \sim N\left(0, \Sigma_{\eta, t}^{*}\right) \\
& E\left(\eta_{t}^{*} \xi_{t}^{\prime}\right)=\overline{L_{t}^{*}} \\
& \Sigma_{\eta, t}^{*}=\Sigma_{\eta, t}-\bar{L} P_{\varepsilon}^{-1} \bar{L}+\bar{L} P_{\varepsilon}^{-1}\left[\left\{P_{|\varepsilon|}-\frac{2}{\pi} l \imath^{\prime}\right\} \mathrm{o}\left(s_{t} s_{t}^{\prime}\right)\right] P_{\varepsilon}^{-1} \bar{L} \\
& \Gamma_{t}^{*}=\sqrt{\frac{2}{\pi}} \bar{L} P_{\varepsilon}^{-1} s_{t} \\
& \bar{L}_{t}^{*}=\bar{L} P_{\varepsilon}^{-1}\left[\left\{R_{|\varepsilon|}-c \sqrt{\frac{2}{\pi}}\right\} \mathrm{o}\left(s_{t} t^{\prime}\right)\right]
\end{aligned}
$$

where $s_{t}$ is a vector constructed from the signs of the returns in $y_{t}$ vector, $c=-1.2703$, and the expressions for $P_{|\varepsilon|}$ and $R_{|\varepsilon|}$ can be found in the appendix of Asai and McAleer (2006). As expected, when the parameter values in $\bar{L}$ matrix are equal to zero in the equations above, the state space form representation of CCMSV is obtained. Using this transformation, it is straightforward to estimate the MSV models with leverage by QML method by using a properly constructed Kalman filtering.

\subsubsection{Monte Carlo Likelihood (MCL) Estimation}

Proposed by Durbin and Koopman (1997) and Shephard and Pitt (1997), this estimation method is based on constructing the likelihood function for general state space models using Monte Carlo techniques. Sandmann and Koopman (1998) put the log-squared transformed returns to a linear non-Gaussian state space form and proceed with the estimation taking into account the true distribution of the log-squared transformed errors. What we refer to as the MCL method in this paper is the method proposed by Jungbacker and Koopman (2006), which extended the method of Durbin and Koopman (1997) for the observation vector without the log-squared transformation. Other simulated maximum likelihood methods are considered by Danielsson and Richard (1993) and Liesenfeld and Richard (2003). In the MCL method, the loglikelihood function is obtained as a sum of a Gaussian part, constructed via Kalman filter, and a minor remainder part, which is evaluated using simulations. Therefore the MCL method only needs a small number of simulations to achieve the desirable accuracy for empirical analysis.

\footnotetext{
${ }^{16}$ Alternatively the $D$ matrix could have been defined as a lower triangular matrix, with nonzero values in the leading diagonal and the intercept term in the volatility equation, $\Gamma$, is a vector of zeros. Then the additional term in the loglikelihood would be different than zero. See Jungbacker and Koopman (2006) for details.
} 
After some manipulations, Durbin and Koopman (1997) showed that the likelihood function for the non-Gaussian model based on importance sampling can be written by:

$$
p(y)=p_{G}(y) \int \frac{p(y \mid h) p(h)}{p_{G}(y \mid h) p_{G}(h)} p_{G}(h \mid y) d h
$$

where $p_{G}(y)$ represents the Gaussian likelihood function of the approximating model, which is defined by:

$$
\tilde{y}_{t}=h_{t}+v_{t}
$$

where $v_{t} \sim N\left(0, G_{t}\right)$ for $t=1, \ldots, N$ and and $h_{t}$ is defined as before. If we would define

$$
\left.\&\left(y_{t} \mid \hat{h}\right)=\frac{\partial \log p\left(y_{t} \mid h_{t}\right)}{\partial h_{t}} \text { and } y_{t} \mid h_{t}\right)=\frac{\partial^{2} \log p\left(y_{t} \mid h_{t}\right)}{\partial h_{t} \partial h_{t}^{\prime}}
$$

then $G_{t}=-\ddot{p}\left(y_{t} \mid \hat{h}_{t}\right)^{-1}$ for $t=1, \ldots, N$ and $\hat{h}$ is the mode of $p(h \mid y)$. In this paper, the models considered have $p(h)=p_{G}(h)$, therefore further simplification can be done on the likelihood.

By defining $\tilde{y}_{t}=\widehat{h}_{t}+G_{t} \dot{p}\left(y_{t} \mid \widehat{h}_{t}\right)$, it can be shown that the first and second derivatives of $\log p(h \mid y)$ and $\log p(h \mid \tilde{y})$ agree in the mode $\hat{h}$. Using the algorithm in Jungbacker and Koopman (2006) based on Kalman filtering and smoothing, one can compute this mode (see Jungbacker and Koopman, 2006, for an illustration with a univariate SV model). Later the Monte Carlo estimator of the likelihood is then given by:

$$
\hat{p}(y)=p_{G}(\tilde{y}) M^{-1} \sum_{m=1}^{M} \omega\left(\alpha^{m}\right)
$$

where

$$
\omega\left(\alpha^{m}\right)=\frac{p(y \mid h)}{p_{G}(\tilde{y} \mid h)} \text { and } \alpha^{m} \sim p_{G}(h \mid \tilde{y})
$$

where $M$ is the number of samples to be generated from $p_{G}(h \mid \tilde{y})$ using the simulation smoother algorithm of Jong and Shephard (1995) or Durbin and Koopman (2002). However, as noted in Jungbacker and Koopman (2005), when $G_{t}=-\ddot{p}\left(y_{t} \mid \hat{h}_{t}\right)^{-1}$ is not positive definite, the simulation smoothing method of Durbin and Koopman (2002) cannot be used. In our estimations we take the number of draws as $M=200$.

In the case of CCMSV model, first and second derivatives $\dot{p}\left(y_{t} \mid h_{t}\right)$ and $\ddot{p}\left(y_{t} \mid h_{t}\right)$ can be obtained from the conditional density:

$$
\log p\left(y_{t} \mid h_{t}\right)=-0.5 k \log (2 \pi)-0.5 \sum_{i=1}^{k} h_{i t}-0.5 \log \left(\operatorname{det}\left(P_{\varepsilon}\right)\right)-0.5 d_{t}^{\prime} P_{\varepsilon}^{-1} d_{t}
$$

for $t=1, \ldots, T$, where $d_{t}=H_{t}^{-1 / 2} y_{t}$. The possible existence of an indefinite matrix for $\ddot{p}\left(y_{t} \mid \hat{h}_{t}\right)$ requires the approach of Jungbacker and Koopman (2005). As Jungbacker and Koopman (2006) suggested, when the model gets too complicated or when explicit expressions for $\dot{p}\left(y_{t} \mid h_{t}\right)$ and $\ddot{p}\left(y_{t} \mid h_{t}\right)$ can not be obtained analytically, as a last resort numerical approximations can be used. For the CCMSV model the analytical derivatives are provided by Jungbacker and Koopman (2006), and these can also be used to obtain the derivatives for TVCMSV. In our estimations, we used analytical derivatives also for the MSV with leverage models, and we provide them in the appendix.

Finally, the in-sample estimates of the underlying volatilities can be obtained from the smoothed estimate of the state vector $\alpha$, (which is just volatilities in the case of the CCMSV and TVCMSV models, but a larger vector in MSV models with leverage) that can be computed from: 


$$
\hat{\alpha}=\frac{\sum_{i=1}^{M} \alpha^{i} \omega\left(\alpha^{i}\right)}{\sum_{i=1}^{M} \alpha^{i}}
$$

where $\alpha^{i}$ is a draw from the conditional density $p_{G}(\alpha \mid y)$ for the approximating Gaussian linear model. When making these draws, the simulation device mentioned in Jungbacker and Koopman (2005) is used to increase the computational efficiency. This device is based on an unconditional draw from $p(\alpha)$ and on a conditional mean adjustment (see Jungbacker and Koopman, 2005, for details).

In our experience, the computational time required for MCL estimation turned out to be high compared to that of the QML estimation. Especially when the sample size or the cross-section size is increased, it takes our code more time to converge than it does for QML estimation. On the other hand, when the cross-section size is large, it is not that obvious to write the analytical derivatives, and if instead one considers numerical derivatives in this case, then the derivatives calculated with respect to large state vectors could be very time consuming and numerically unstable (see also Jungbacker and Koopman, 2006). Meanwhile, QML method is computationally more convenient and also more flexible for considering a higher cross-section size.

\section{MONTE CARLO EXPERIMENTS}

In this section, we report the results of our Monte Carlo (MC) experiments. Our aim is to compare the performance of QML and MCL methods when estimating the models considered in this paper for several different parameter sets. For each model and parameter set, we generated $B=500$ bivariate time series vectors of dimension with sample size $T=500 .{ }^{17}$ For comparison purposes, we look at the performances in parameter estimation as well as in insample smoothed volatility and correlation estimations. The estimation results are reported in terms of MC means of parameter estimates, corresponding MC standard deviations and root mean squared error for each parameter estimate as a measure of efficiency ${ }^{18}$. Moreover, we provide figures of kernel density estimates of the mean deviations and mean absolute deviations of estimated log-volatilities, $\widehat{h}_{i t}$, and correlations, $\hat{p}_{t}$, from their true values ${ }^{19}$. These deviations are calculated for each series over the number of simulations $B$; i.e. for each $t$ :

$$
\begin{gathered}
\Delta \hat{h}_{i t}=\frac{1}{B} \sum_{b=1}^{B}\left(\hat{h}_{i t, b}-h_{i t, b}\right) \text { and }|\Delta| \hat{h}_{i t}=\frac{1}{B} \sum_{b=1}^{B}\left|\hat{h}_{i t, b}-h_{i t, b}\right| \\
\Delta \hat{p}_{t}=\frac{1}{B} \sum_{b=1}^{B}\left(\frac{\hat{p}_{t, b}-p_{t, b}}{p_{t, b}}\right) \text { and }|\Delta| \hat{p}_{t}=\frac{1}{B} \sum_{b=1}^{B}\left|\frac{\hat{p}_{t, b}-p_{t, b} \mid}{p_{t, b}}\right|
\end{gathered}
$$

where |.| is an absolute value. Given that in the case of Constant Correlation MSV (CCMSV) and MSV models with leverage the correlations are constant (the correlation estimate is actually a parameter estimate), the kernel density estimate of the deviations of $B$ different estimates of the correlation parameter from the true correlation parameter will be plotted. However, for the Time Varying Correlation MSV (TVCMSV), as in the case of volatilities, the kernel density

\footnotetext{
${ }^{17}$ We also consider a trivariate time series vector and sample sizes $T=200$ and $T=1000$ with the parameter set of the first experiment of the CCMSV model.

${ }^{18}$ For comparison purposes, in case of MSV with leverage models as well we report the results for the parameters in $P_{\varepsilon}$ and $Q_{\eta}$ matrices, instead of reporting the results for the Cholesky factors in their formulation (see section $\$ 2.3 \$$ ).

${ }^{19}$ It should be noted that $\hat{h}_{i t, b}-h_{i t, b}$, is a log-difference, while $\hat{p}_{t, b}-p_{t, b}$ is only a difference. Therefore the latter is divided by $p_{t, b}$ to have the same sense of percentage deviation.
} 
estimates of $\Delta \hat{p}_{t}$ and $\left|\Delta \hat{p}_{t}\right|$ are plotted. Finally, mean absolute errors (MAE) and root mean squared errors (RMSE) of the volatility and correlation estimates are also reported. In this case, the MAE and the RMSE are calculated as:

$$
\begin{gathered}
M A E=\frac{1}{T} \frac{1}{B} \sum_{t=1}^{T} \sum_{b=1}^{B}\left|\hat{h}_{i t, b}-h_{i t, b}\right| \text { and } R M S E=\sqrt{\frac{1}{T} \frac{1}{B} \sum_{t=1}^{T} \sum_{b=1}^{B}\left(\hat{h}_{i t, b}-h_{i t, b}\right)^{2}} \\
M A E=\frac{1}{T} \frac{1}{B} \sum_{t=1}^{T} \sum_{b=1}^{B}\left|\frac{\hat{p}_{t, b}-p_{t, b} \mid}{p_{t, b}}\right| \text { and } R M S E=\sqrt{\frac{1}{T} \frac{1}{B} \sum_{t=1}^{T} \sum_{b=1}^{B}\left(\frac{\hat{p}_{t, b}-p_{t, b}}{p_{t, b}}\right)^{2}}
\end{gathered}
$$

\begin{tabular}{|c|c|c|c|c|c|c|c|c|}
\hline Estim.IParam. & $\left\{P_{\varepsilon}\right\}_{21}$ & $\Gamma_{11}$ & $\Gamma_{21}$ & $\Phi_{11}$ & $\Phi_{22}$ & $\left\{Q_{\eta}\right\}_{11}$ & $\left\{Q_{\eta}\right\}_{21}$ & $\left\{Q_{\eta}\right\}_{22}$ \\
\hline Exp $1 / T=200$, True & 0.2000 & -0.1000 & -0.1300 & 0.9000 & 0.9500 & 0.1500 & 0.0400 & 0.0800 \\
\hline \multirow[t]{3}{*}{ QML } & 0.2589 & -0.2080 & -0.2819 & 0.8031 & 0.8938 & 0.3126 & 0.0505 & 0.1736 \\
\hline & $(0.2288)$ & $(0.2670)$ & $(0.4039)$ & $(0.2114)$ & $(0.1395)$ & $(0.4733)$ & $(0.1094)$ & $(0.3725)$ \\
\hline & [0.2363] & [0.2880] & [0.4315] & [0.2325] & [0.1504] & [0.5004] & [0.1099] & [0.3841] \\
\hline \multirow[t]{3}{*}{ MCL } & 0.2011 & -0.2074 & -0.2872 & 0.8023 & 0.8908 & 0.2297 & 0.0558 & 0.1192 \\
\hline & $(0.0710)$ & $(0.2247)$ & $(0.3268)$ & (0.1908) & $(0.1212)$ & $(0.1734)$ & $(0.0668)$ & $(0.1100)$ \\
\hline & [0.0710] & [0.2490] & [0.3626] & [0.2143] & [0.1349] & [0.1909] & {$[0.0686]$} & [0.1168] \\
\hline Exp 1, True & 0.2000 & -0.1000 & -0.1300 & 0.9000 & 0.9500 & 0.1500 & 0.0400 & 0.0800 \\
\hline \multirow[t]{3}{*}{ QML } & 0.1815 & -0.1527 & -0.2189 & 0.8478 & 0.9164 & 0.2425 & 0.0459 & 0.1327 \\
\hline & $(0.1804)$ & $(0.1496)$ & $(0.2911)$ & $(0.1348)$ & $(0.1050)$ & $(0.2880)$ & $(0.0596)$ & $(0.1881)$ \\
\hline & [0.1814] & [0.1586] & [0.3043] & [0.1445] & [0.1102] & [0.3025] & {$[0.0599]$} & [0.1953] \\
\hline \multirow[t]{3}{*}{ MCL } & 0.1955 & -0.1249 & -0.1654 & 0.8754 & 0.9361 & 0.1663 & 0.0409 & 0.0869 \\
\hline & $(0.0454)$ & $(0.0608)$ & $(0.0845)$ & $(0.0519)$ & $(0.0319)$ & $(0.0669)$ & $(0.0271)$ & $(0.0399)$ \\
\hline & [0.0456] & [0.0657] & [0.0916] & {$[0.0574]$} & [0.0348] & [0.0689] & [0.0271] & [0.0405] \\
\hline Exp $1 / T=1000$, True & 0.2000 & -0.1000 & -0.1300 & 0.9000 & 0.9500 & 0.1500 & 0.0400 & 0.0800 \\
\hline \multirow[t]{3}{*}{ QML } & 0.1712 & -0.1245 & -0.1611 & 0.8751 & 0.9383 & 0.1933 & 0.0457 & 0.0979 \\
\hline & $(0.1545)$ & $(0.0802)$ & $(0.0905)$ & $(0.0741)$ & $(0.0338)$ & $(0.1566)$ & $(0.0304)$ & $(0.0639)$ \\
\hline & [0.1572] & [0.0838] & [0.0957] & {$[0.0782]$} & [0.0357] & [0.1624] & [0.0309] & [0.0664] \\
\hline \multirow[t]{3}{*}{ MCL } & 0.1991 & -0.1137 & -0.1493 & 0.8853 & 0.9426 & 0.1587 & 0.0415 & 0.0844 \\
\hline & $(0.0313)$ & $(0.0376)$ & $(0.0495)$ & $(0.0343)$ & $(0.0187)$ & $(0.0472)$ & $(0.0176)$ & $(0.0245)$ \\
\hline & [0.0313] & [0.0401] & [0.0532] & {$[0.0373]$} & [0.0201] & [0.0480] & {$[0.0176]$} & [0.0249] \\
\hline Exp 2, True & 0.2000 & -0.1000 & -0.1300 & 0.9000 & 0.9800 & 0.1500 & 0.0400 & 0.0800 \\
\hline \multirow[t]{3}{*}{ QML } & 0.1799 & -0.1608 & -0.2323 & 0.8411 & 0.9643 & 0.2425 & 0.0438 & 0.0992 \\
\hline & $(0.1848)$ & $(0.1572)$ & $(0.3096)$ & $(0.1430)$ & $(0.0473)$ & $(0.2837)$ & $(0.0485)$ & $(0.0882)$ \\
\hline & [0.1859] & [0.1686] & [0.3261] & [0.1546] & [0.0499] & [0.2984] & [0.0486] & [0.0902] \\
\hline \multirow[t]{3}{*}{ MCL } & 0.2007 & -0.1305 & -0.2015 & 0.8704 & 0.9690 & 0.1720 & 0.0435 & 0.0852 \\
\hline & $(0.0435)$ & $(0.0670)$ & $(0.1225)$ & $(0.0616)$ & (0.0188) & $(0.0752)$ & $(0.0288)$ & $(0.0326)$ \\
\hline & [0.0435] & [0.0736] & [0.1418] & {$[0.0684]$} & [0.0218] & [0.0784] & {$[0.0291]$} & [0.0330] \\
\hline Exp 3, True & 0.2000 & -0.1000 & -0.1300 & 0.9000 & 0.9500 & 0.4000 & 0.1500 & 0.3500 \\
\hline \multirow[t]{3}{*}{ QML } & 0.1923 & -0.1244 & -0.1575 & 0.8754 & 0.9388 & 0.4748 & 0.1573 & 0.3733 \\
\hline & $(0.1784)$ & $(0.0759)$ & $(0.0856)$ & $(0.0555)$ & $(0.0277)$ & $(0.2547)$ & $(0.0749)$ & (0.1573) \\
\hline & [0.1785] & [0.0797] & [0.0899] & {$[0.0607]$} & [0.0299] & [0.2655] & [0.0753] & [0.1590] \\
\hline \multirow[t]{3}{*}{ MCL } & 0.2003 & -0.1110 & -0.1461 & 0.8878 & 0.9429 & 0.3927 & 0.1452 & 0.3374 \\
\hline & $(0.0469)$ & $(0.0506)$ & $(0.0574)$ & $(0.0349)$ & $(0.0191)$ & (0.0979) & $(0.0539)$ & $(0.0820)$ \\
\hline & {$[0.0469]$} & [0.0518] & {$[0.0597]$} & {$[0.0370]$} & [0.0204] & [0.0981] & {$[0.0541]$} & [0.0829] \\
\hline
\end{tabular}

Table 3.1 The parameter estimation results of the simulations where the data is generated by a CC-MSV model and estimated via QML and MCL methods.

Notes: The sample size is $T=500$, if not noted otherwise. For each experiment, the true parameter values are reported in the first row. Then for each estimation method, MC mean, standard deviation (in parantheses) and root mean squared error (in square brackets) of the parameter estimates are reported, respectively. Experiments 1-3.

For the CCMSV model, the true values of $\Psi=\left(\operatorname{vecl}\left(P_{\varepsilon}\right)^{\prime}, \Gamma^{\prime}, \operatorname{diag}(\Phi)^{\prime}, \operatorname{vech}\left(Q_{\eta}\right)^{\prime}\right)^{\prime}$ parameters are chosen close to the ones in Harvey et al. (1994) and Jungbacker and Koopman (2006). The true values of the parameters and the parameter estimation results are given in Tables 3.1 and 3.2. These results for the CCMSV model confirm the previous results in the literature that the finite sample performance of MCL is better than that of QML; i.e. the QML method is less efficient. The kernel density estimates of the mean and absolute mean deviations are plotted in Figures 3.1-3.4. Finally, the MAE's and RMSE's of the volatility and correlation estimates are given in Table 3.3. We can summarize the results in these tables and figures as follows. For the first experiment (Exp. 1) of CCMSV model, increasing the sample size from $T=200$ to $T=500$ and then to $T=1000$ increases the efficiency of both QML and MCL parameter estimators, while it does less so for the QML method. Similarly, the MAE's and RMSE's of the deviations from 
true volatilities and correlations improve for both methods. This can be confirmed from Figure 3.1 .

\begin{tabular}{|c|c|c|c|c|c|c|c|c|}
\hline Estim.IParam. & $\left\{P_{\varepsilon}\right\}_{21}$ & $\Gamma_{11}$ & $\Gamma_{21}$ & $\Phi_{11}$ & $\Phi_{22}$ & $\left\{Q_{\eta}\right\}_{11}$ & $\left\{Q_{\eta}\right\}_{21}$ & $\left\{Q_{\eta}\right\}_{22}$ \\
\hline Exp 4 - True & 0.2000 & -0.1000 & -0.1300 & 0.9000 & 0.9800 & 0.4000 & 0.1500 & 0.3500 \\
\hline \multirow[t]{3}{*}{ QML } & 0.1933 & -0.1244 & -0.1937 & 0.8755 & 0.9702 & 0.4637 & 0.1509 & 0.3590 \\
\hline & $(0.1854)$ & $(0.0713)$ & $(0.1087)$ & $(0.0552)$ & $(0.0161)$ & $(0.2416)$ & $(0.0773)$ & (0.1149) \\
\hline & [0.1855] & [0.0753] & {$[0.1260]$} & {$[0.0604]$} & [0.0188] & [0.2499] & [0.0773] & [0.1153] \\
\hline \multirow[t]{3}{*}{ MCL } & 0.1989 & -0.1146 & -0.18451 & 0.8850 & 0.9716 & 0.3976 & 0.1493 & 0.3436 \\
\hline & $(0.0443)$ & $(0.0492)$ & $(0.0938)$ & $(0.0350)$ & $(0.0134)$ & $(0.0968)$ & $(0.0567)$ & $(0.0766)$ \\
\hline & {$[0.0443]$} & [0.0514] & [0.1088] & {$[0.0381]$} & [0.0158] & {$[0.0968]$} & {$[0.0567]$} & {$[0.0769]$} \\
\hline Exp 5-True & 0.8000 & -0.1000 & $\begin{array}{l}-0.1300 \\
\end{array}$ & 0.9000 & 0.9500 & 0.1500 & 0.0400 & 0.0800 \\
\hline \multirow{3}{*}{ QML } & 0.8038 & -0.1677 & -0.2246 & 0.8361 & 0.9149 & 0.2706 & 0.0585 & 0.1372 \\
\hline & $(0.0408)$ & $(0.1510)$ & $(0.2631)$ & $(0.1342)$ & $(0.0951)$ & $(0.2873)$ & $(0.0666)$ & $(0.1842)$ \\
\hline & [0.0410] & [0.1655] & [0.2796] & [0.1486] & [0.1014] & [0.3116] & {$[0.0691]$} & [0.1928] \\
\hline \multirow[t]{3}{*}{ MCL } & 0.7980 & -0.1231 & -0.1786 & 0.8769 & 0.9324 & 0.1630 & 0.0467 & 0.0972 \\
\hline & $(0.0183)$ & $(0.0598)$ & $(0.2028)$ & (0.0515) & $(0.0670)$ & $(0.0834)$ & $(0.0450)$ & $(0.1654)$ \\
\hline & {$[0.0184]$} & [0.0641] & {$[0.2085]$} & {$[0.0565]$} & [0.0693] & {$[0.0844]$} & {$[0.0455]$} & {$[0.1663]$} \\
\hline Exp 6-True & 0.8000 & -0.1000 & -0.1300 & 0.9000 & 0.9800 & 0.4000 & 0.1500 & 0.3500 \\
\hline \multirow[t]{3}{*}{ QML } & 0.8048 & -0.1304 & -0.1856 & 0.8717 & 0.9711 & 0.4860 & 0.1658 & 0.3601 \\
\hline & $(0.0422)$ & (0.0749) & $(0.1025)$ & $(0.0561)$ & $(0.0152)$ & $(0.2275)$ & $(0.0874)$ & (0.1038) \\
\hline & [0.0424] & [0.0808] & {$[0.1166]$} & {$[0.0629]$} & [0.0176] & {$[0.2432]$} & [0.0888] & [0.1043] \\
\hline \multirow[t]{3}{*}{ MCL } & 0.7782 & -0.1162 & -0.1722 & 0.8873 & 0.9735 & 0.3880 & 0.1691 & 0.3385 \\
\hline & $(0.0360)$ & (0.0496) & (0.0878) & $(0.0325)$ & $(0.0125)$ & $(0.1209)$ & $(0.0766)$ & $(0.0759)$ \\
\hline & {$[0.0421]$} & {$[0.0522]$} & {$[0.0974]$} & {$[0.0349]$} & [0.0141] & {$[0.1215]$} & {$[0.0789]$} & [0.0768] \\
\hline Exp 7 - True & 0.8000 & -0.1000 & -0.1300 & 0.9000 & 0.9500 & 0.4000 & 0.1500 & 0.3500 \\
\hline \multirow{3}{*}{ QML } & 0.8042 & -0.1236 & -0.1655 & 0.8768 & 0.9366 & 0.4609 & 0.1585 & 0.3837 \\
\hline & $(0.0433)$ & $(0.0693)$ & (0.0864) & $(0.0561)$ & $(0.0296)$ & $(0.2321)$ & $(0.0909)$ & $(0.1509)$ \\
\hline & {$[0.0435]$} & [0.0732] & {$[0.0934]$} & [0.0607] & [0.0325] & [0.2399] & [0.0913] & [0.1546] \\
\hline \multirow[t]{3}{*}{ MCL } & 0.7790 & -0.1124 & -0.1485 & 0.8903 & 0.9438 & 0.3762 & 0.1581 & 0.3365 \\
\hline & $(0.0320)$ & $(0.0553)$ & $(0.0754)$ & $(0.0415)$ & $(0.0239)$ & $(0.1086)$ & $(0.0870)$ & $(0.1186)$ \\
\hline & [0.0382] & {$[0.0566]$} & {$[0.0777]$} & {$[0.0426]$} & {$[0.0247]$} & [0.1111] & {$[0.0874]$} & [0.1193] \\
\hline Exp 8- & 0.8000 & -0.1000 & -0.1300 & 0.9000 & 0.9800 & 0.1500 & 0.0400 & 0.0800 \\
\hline \multirow[t]{3}{*}{ QML } & 0.8069 & -0.1661 & -0.2187 & 0.8362 & 0.9662 & 0.2669 & 0.0493 & 0.0991 \\
\hline & $(0.0412)$ & $(0.1706)$ & $(0.1946)$ & $(0.1539)$ & $(0.0305)$ & $(0.3193)$ & $(0.0492)$ & $(0.0734)$ \\
\hline & [0.0417] & [0.1830] & {$[0.2139]$} & {$[0.1666]$} & [0.0335] & {$[0.3400]$} & {$[0.0501]$} & {$[0.0758]$} \\
\hline \multirow[t]{3}{*}{ MCL } & 0.7966 & -0.1189 & -0.1819 & 0.8804 & 0.9719 & 0.1556 & 0.0449 & 0.0827 \\
\hline & $(0.0174)$ & $(0.0520)$ & $(0.0954)$ & $(0.0452)$ & $(0.0145)$ & $(0.0636)$ & $(0.0268)$ & $(0.0277)$ \\
\hline & {$[0.0178]$} & {$[0.0553]$} & {$[0.1086]$} & {$[0.0493]$} & {$[0.0166]$} & {$[0.0638]$} & {$[0.0273]$} & {$[0.0278]$} \\
\hline
\end{tabular}

Table 3.2 The parameter estimation results of the simulations where the data is generated by a CC-MSV model and estimated via QML and MCL methods.

Notes: The sample size is $T=500$, if not noted otherwise. For each experiment, the true parameter values are reported in the first row. Then for each estimation method, MC mean, standard deviation (in parantheses) and root mean squared error (in square brackets) of the parameter estimates are reported, respectively. Experiments 4-8.

We can see in Table 3.3 that while the ratio of MAE's and RMSE's volatility estimates of QML to that of MCL are more or less similar across these sample sizes, the efficiency of the QML estimator of correlation parameter improves relatively less compared to that of the MCL estimator. We also performed a trivariate experiment as an extension to the first experiment. We chose the parameter values for the third series similar to the first two series and considered a sample size of $T=500$. The results that we obtained are similar, hence are not reported here but are available from the author upon request.

We can conclude that the efficiency of QML and MCL estimators of the correlation parameter increases as the two series become more correlated. ( $p=0.2$ vs $p=0.8$; see experiments 1 vs 5 , 2 vs 8,3 vs 7 and 4 vs 6). However, this conclusion does not hold for all the parameters. Also the QML/MCL ratio of MAE's and RMSE's of the deviations from true correlations decreases, implying that QML estimator of the correlation parameter improves relatively more compared to MCL estimator. When the series are less correlated $(p=0.2)$, the QML doesn't estimate the correlation parameter very accurately; even though the mean is more or less around the true value, we observe a relatively high variance. This can be confirmed visually from the third columns of Figures 3.1-3.4. 
When the second autoregressive parameter, $\Phi_{22}$, is higher we observe that the RMSE of the QML and MCL estimates of $\Phi_{22}$ and $Q_{22}$ are less. $\left(\Phi_{22}=0.95\right.$ vs $\Phi_{22}=0.98$; see experiments 1 vs 2, 5 vs 8, 3 vs 4 and 6 vs 7). We also observed that the QML estimator of the correlation parameter is performing slightly worse.

\begin{tabular}{|c|c|c|c|c|c|c|c|c|c|}
\hline MAE & Method & $|\Delta| h_{1 t}$ & $|\Delta| h_{2 t}$ & $|\Delta| p$ & RMSE & Method & $\Delta h_{1 t}$ & $\Delta h_{2 t}$ & $\Delta p$ \\
\hline Exp1 & QML & 0.5584 & 0.4968 & 0.2080 & Exp1 & QML & 0.7113 & 0.6321 & 0.2363 \\
\hline \multirow[t]{2}{*}{$T=200$} & MCL & 0.4821 & 0.4164 & 0.0576 & $\mathrm{~T}=200$ & MCL & 0.6098 & 0.5286 & 0.0710 \\
\hline & QML/MCL & 1.1582 & 1.1931 & 3.6129 & & QML/MCL & 1.1665 & 1.1959 & 3.3267 \\
\hline Exp1 & QML & 0.5292 & 0.4655 & 0.1632 & Exp1 & QML & 0.6693 & 0.5883 & 0.1814 \\
\hline \multirow[t]{2}{*}{$\mathrm{T}=500$} & MCL & 0.4529 & 0.3903 & 0.0355 & $\mathrm{~T}=500$ & MCL & 0.5701 & 0.4925 & 0.0456 \\
\hline & QML/MCL & 1.1685 & 1.1928 & 4.5959 & & QML/MCL & 1.1740 & 1.1947 & 3.9749 \\
\hline Exp1 & QML & 0.5141 & 0.4470 & 0.1402 & Exp1 & QML & 0.6470 & 0.5626 & 0.1572 \\
\hline \multirow[t]{2}{*}{$\mathrm{T}=1000$} & MCL & 0.4487 & 0.3816 & 0.0254 & $\mathrm{~T}=1000$ & MCL & 0.5644 & 0.4809 & 0.0313 \\
\hline & QML/MCL & 1.1458 & 1.1716 & 5.5240 & & QML/MCL & 1.1463 & 1.1699 & 5.0245 \\
\hline \multirow[t]{3}{*}{ Exp2 } & QML & 0.5276 & 0.4932 & 0.1698 & Exp2 & QML & 0.6669 & 0.6315 & 0.1859 \\
\hline & MCL & 0.4536 & 0.4275 & 0.0351 & & MCL & 0.5710 & 0.5560 & 0.0435 \\
\hline & QML/MCL & 1.1632 & 1.1539 & 4.8401 & & QML/MCL & 1.1679 & 1.1358 & 4.2731 \\
\hline \multirow[t]{3}{*}{ Exp3 } & QML & 0.6941 & 0.6841 & 0.1618 & Exp3 & QML & 0.8755 & 0.8680 & 0.1785 \\
\hline & MCL & 0.6209 & 0.6134 & 0.0374 & & MCL & 0.7834 & 0.7850 & 0.0469 \\
\hline & QML/MCL & 1.1179 & 1.1153 & 4.3253 & & QML/MCL & 1.1176 & 1.1057 & 3.8052 \\
\hline \multirow[t]{3}{*}{ Exp4 } & QML & 0.6916 & 0.7483 & 0.1679 & Exp4 & QML & 0.8717 & 0.9899 & 0.1855 \\
\hline & MCL & 0.6206 & 0.6996 & 0.0354 & & MCL & 0.7822 & 0.9468 & 0.0443 \\
\hline & QML/MCL & 1.1144 & 1.0697 & 4.7385 & & QML/MCL & 1.1144 & 1.0456 & 4.1828 \\
\hline \multirow[t]{3}{*}{ Exp5 } & QML & 0.5307 & 0.4682 & 0.0329 & Exp5 & QML & 0.6718 & 0.5933 & 0.0410 \\
\hline & MCL & 0.4322 & 0.3749 & 0.0144 & & MCL & 0.5456 & 0.4804 & 0.0184 \\
\hline & QML/MCL & 1.2280 & 1.2482 & 2.2885 & & QML/MCL & 1.2313 & 1.2350 & 2.2326 \\
\hline \multirow[t]{3}{*}{ Exp6 } & QML & 0.6966 & 0.7587 & 0.0338 & Exp6 & QML & 0.8798 & 1.0041 & 0.0424 \\
\hline & MCL & 0.6027 & 0.7149 & 0.0270 & & MCL & 0.7626 & 0.9955 & 0.0421 \\
\hline & QML/MCL & 1.1559 & 1.0612 & 1.2516 & & QML/MCL & 1.1538 & 1.0086 & 1.0089 \\
\hline \multirow[t]{3}{*}{ Exp7 } & QML & 0.6964 & 0.6894 & 0.0353 & Exp7 & QML & 0.8835 & 0.8736 & 0.0435 \\
\hline & MCL & 0.6006 & 0.6021 & 0.0258 & & MCL & 0.7587 & 0.7675 & 0.0382 \\
\hline & QML/MCL & 1.1645 & 1.1450 & 1.3679 & & QML/MCL & 1.1645 & 1.1382 & 1.1377 \\
\hline \multirow[t]{3}{*}{ Exp8 } & QML & 0.5330 & 0.5024 & 0.0339 & Exp8 & QML & 0.6756 & 0,6442 & 0.0417 \\
\hline & MCL & 0.4316 & 0.4211 & 0.0142 & & MCL & 0.5432 & 0.5523 & 0.0178 \\
\hline & QML/MCL & 1.2349 & 1.1930 & 2.3861 & & QML/MCL & 1.2438 & 1.1665 & 2.3471 \\
\hline
\end{tabular}

Table 3.3 Mean absolute error (MAE) and root mean squared error (RMSE) of the QML and MCL volatility and correlation estimates for CC-MSV model.

Figure 3.1 Kernel density estimates of the deviations of MCL and QML volatility and correlation estimates from the true ones, for the CCMSV model. Experiments 1 to 4. 
Eratalay-Estimation of Multivariate Stochastic Volatility Models: A Comparative Monte Carlo Study
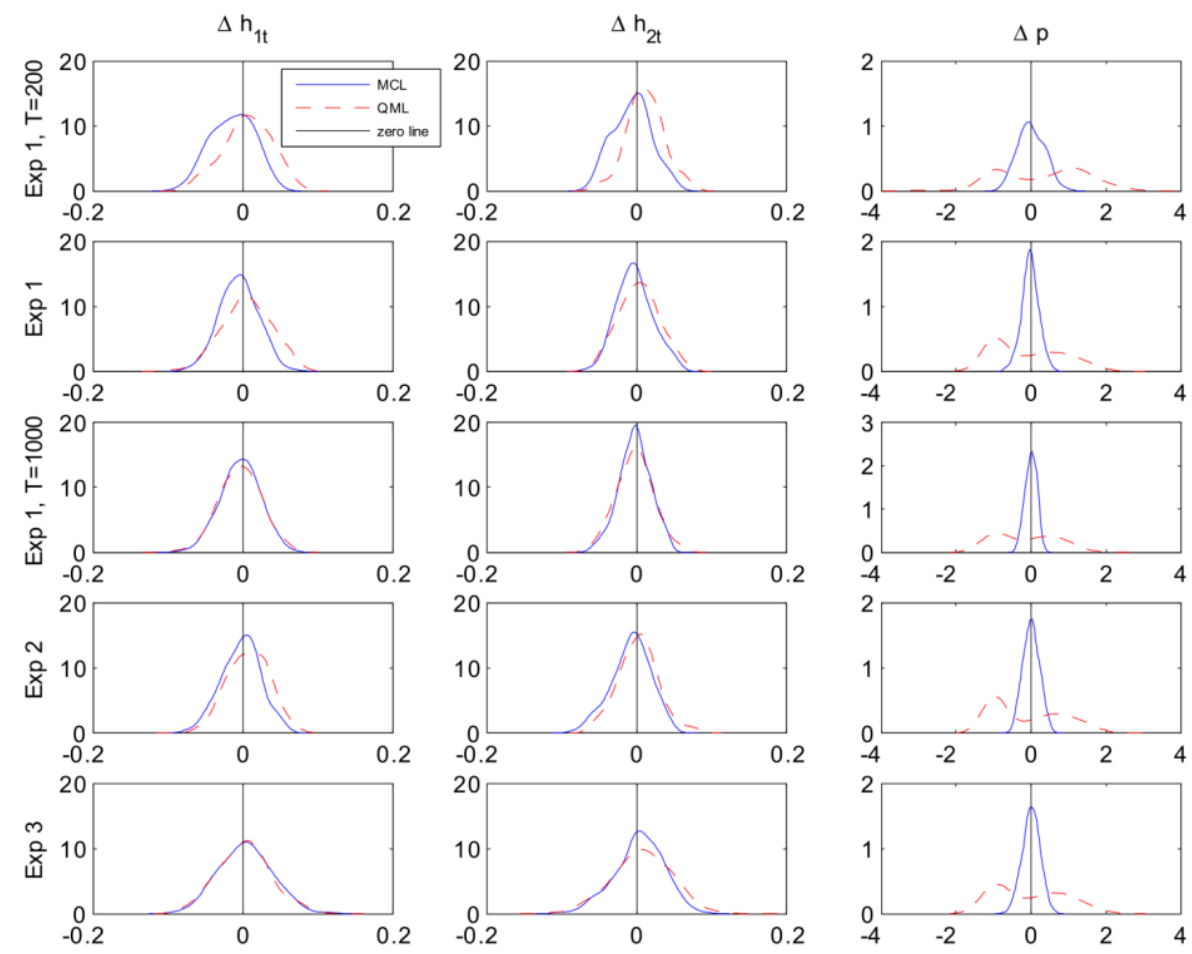

Figure 3.2 Kernel density estimates of the absolute deviations of MCL and QML volatility and correlation estimates from the true ones, for the CCMSV model. Experiments 1 to 4.
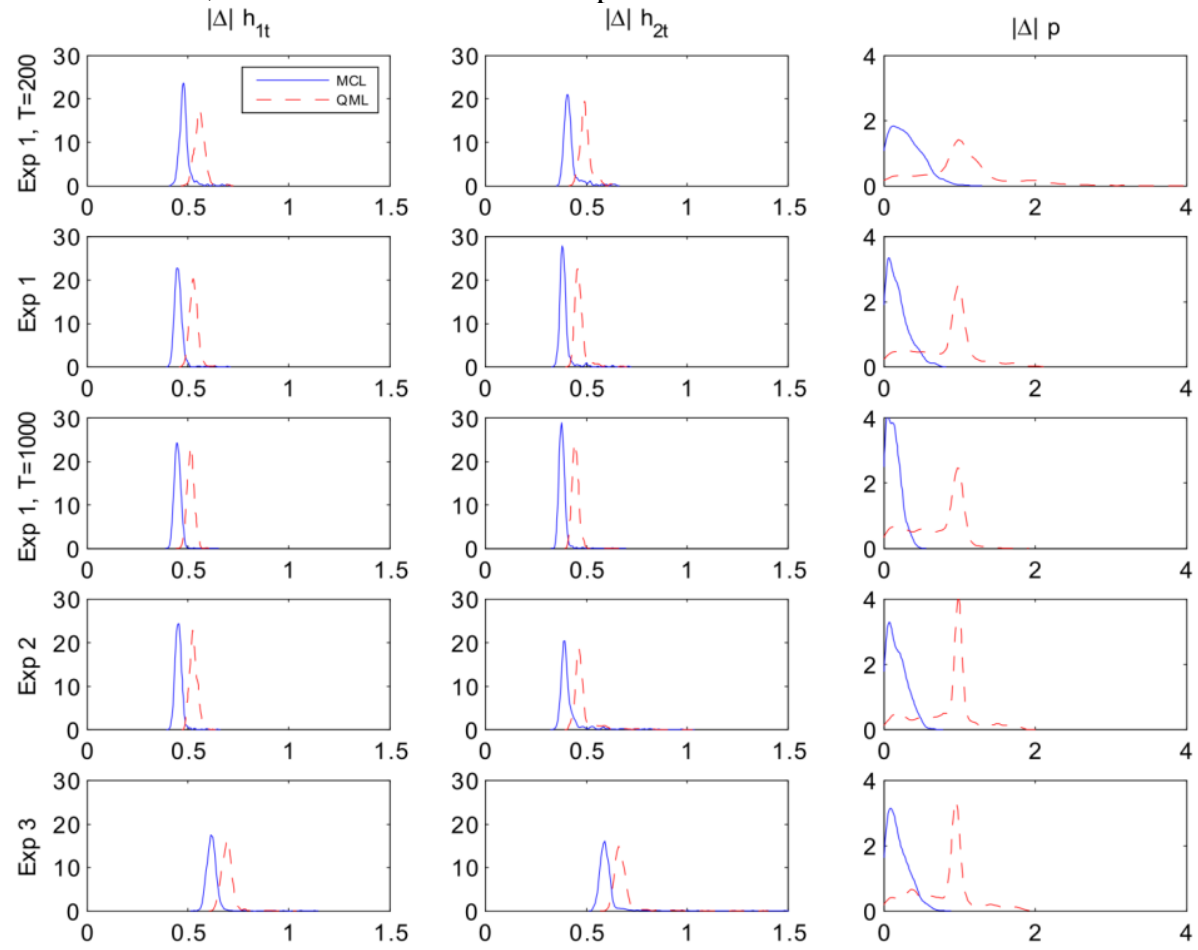

Figure 3.3 Kernel density estimates of the deviations of MCL and QML volatility and correlation estimates from the true ones, for the CCMSV model. Experiments 5 to 8. 

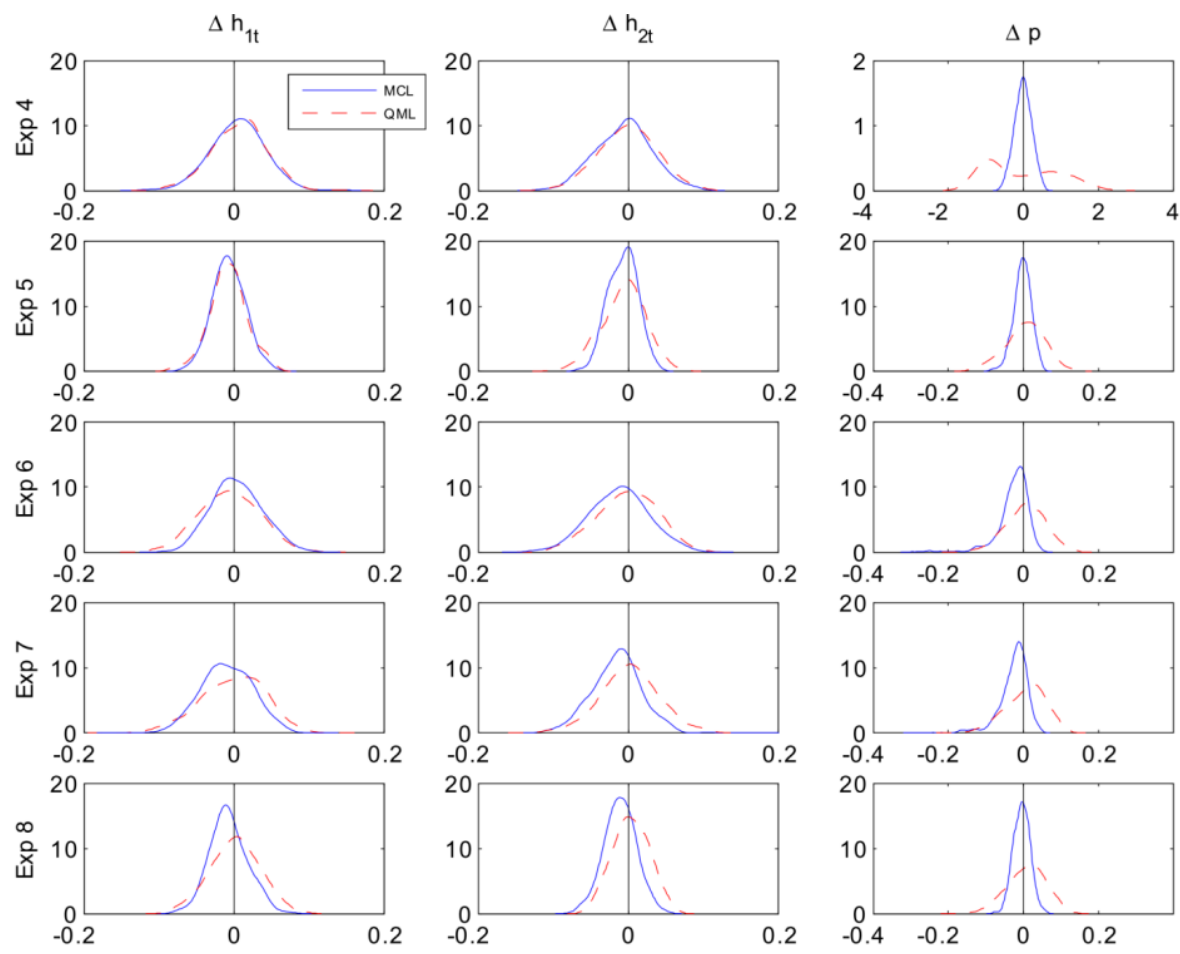

Figure 3.4 Kernel density estimates of the absolute deviations of MCL and QML volatility and correlation estimates from the true ones, for the CCMSV model. Experiments 5 to 8 .
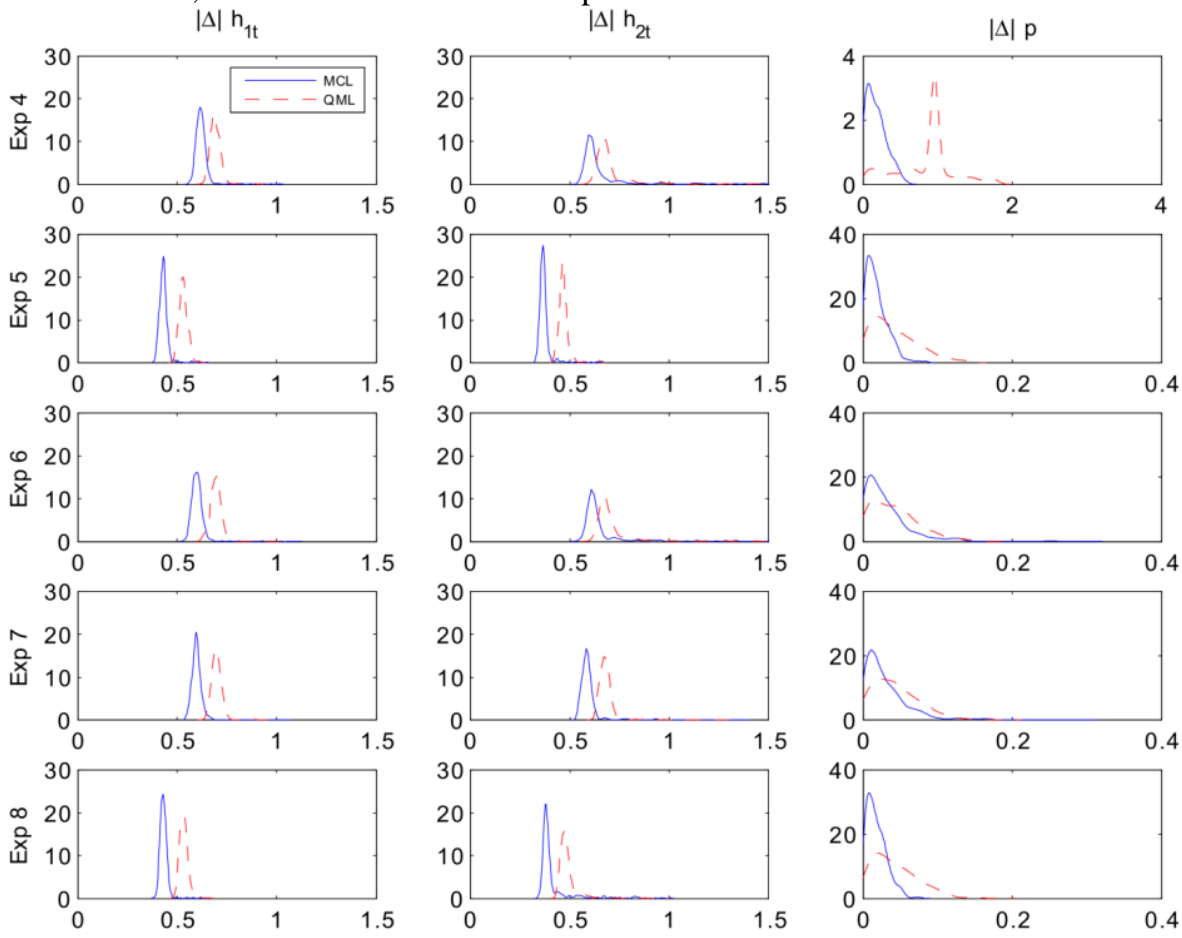

When the variance of the SV processes, the parameters in $Q$, are higher we see that the efficiencies of QML and MCL estimators of correlation parameter do not change much. (See experiments 1 vs 3, 2 vs 4, 5 vs 7 and 6 vs 8) The RMSE's of QML and MCL estimators of many parameters are lower. QML and MCL estimators gain efficiency in estimating the intercept $\$$ Gamma $\$$ and the autoregressive coefficients $\Phi$. For both QML and MCL estimators, the higher variance in the SV processes result in higher deviations from the true volatilities. On the other hand the QML/MCL ratio of the MAE's and RMSE's of the deviations from true volatilities and correlations improve. It is also observed that when $p=0.8$, the higher variance in the SV process affects the efficiency of the MCL correlation estimator negatively. (See 
experiments 5 vs 7 and 6 vs 8). We observe that when $\Phi_{22}=0.98$ and the variance of the SV processes is high, the QML/MCL ratio of the deviations from true volatilities is closer to 1. (See experiment 4 and 6). If the underlying correlation parameter is also high, $p=0.8$, the QML/MCL ratio of the deviations from true correlation is the smallest among all experiments. (See experiment 6).

\begin{tabular}{lcccccccc}
\hline \hline Estim.IParam. & $\{D\}_{21}$ & $\Gamma_{11}$ & $\Gamma_{21}$ & $\Phi_{11}$ & $\Phi_{22}$ & $\left\{Q_{\eta}\right\}_{11}$ & $\left\{Q_{\eta}\right\}_{21}$ & $\left\{Q_{\eta}\right\}_{22}$ \\
\hline Exp 1 - True & 0.2041 & -0.1000 & -0.1300 & 0.9000 & 0.9500 & 0.1500 & 0.0400 & 0.0800 \\
QML & 0.2035 & -0.1664 & -0.2037 & 0.8378 & 0.9215 & 0.2614 & 0.0563 & 0.1129 \\
& $(0.0208)$ & $(0.1751)$ & $(0.2679)$ & $(0.1542)$ & $(0.1004)$ & $(0.2945)$ & $(0.0611)$ & $(0.1542)$ \\
& {$[0.0208]$} & {$[0.1873]$} & {$[0.2779]$} & {$[0.1663]$} & {$[0.1044]$} & {$[0.3149]$} & {$[0.0632]$} & {$[0.1577]$} \\
MCL & 0.2038 & -0.1313 & -0.1718 & 0.8693 & 0.9338 & 0.1752 & 0.0445 & 0.0901 \\
& $(0.0167)$ & $(0.0738)$ & $(0.0996)$ & $(0.0675)$ & $(0.0376)$ & $(0.0839)$ & $(0.0311)$ & $(0.0434)$ \\
& {$[0.0167]$} & {$[0.0802]$} & {$[0.1080]$} & {$[0.0742]$} & {$[0.0409]$} & {$[0.0876]$} & {$[0.0314]$} & {$[0.0445]$} \\
\hline Exp 2 - True & 1.3333 & -0.1000 & -0.1300 & 0.9000 & 0.9500 & 0.1500 & 0.0400 & 0.0800 \\
QML & 1.3357 & -0.1633 & -0.2130 & 0.8411 & 0.9187 & 0.2621 & 0.0569 & 0.1155 \\
& $(0.0197)$ & $(0.1724)$ & $(0.2750)$ & $(0.1478)$ & $(0.1008)$ & $(0.3097)$ & $(0.0636)$ & $(0.1499)$ \\
MCL & {$[0.0198]$} & {$[0.1836]$} & {$[0.2872]$} & {$[0.1591]$} & {$[0.1056]$} & {$[0.3294]$} & {$[0.0658]$} & {$[0.1541]$} \\
& 1.3320 & -0.1299 & -0.1744 & 0.8687 & 0.9332 & 0.1753 & 0.0434 & 0.0908 \\
& $(0.0133)$ & $(0.0725)$ & $(0.0983)$ & $(0.0699)$ & $(0.0381)$ & $(0.0969)$ & $(0.0286)$ & $(0.0442)$ \\
\hline \hline
\end{tabular}

Table 3.4 The parameter estimation results of the simulations where the data is generated by a TVC-MSV model and estimated via QML and MCL methods..

Notes: For each experiment, the true parameter values are reported in the first row. Then for each estimation method, MC mean, standard deviation (in paranthesis) and root mean squared error (in square brackets) of the parameter estimates are reported, respectively.

For the experiments with the TVCMSV model, the true values for the parameters (except the correlation parameter) are chosen from experiment 1 of the CCMSV model. Then two experiments are performed where the correlation parameter values of 0.2041 and 1.3333 are chosen, such that the correlation between the volatility adjusted returns are 0.2 and 0.8 , respectively. The parameter estimation results are given in Table 3.4, the kernel density estimates of the mean and mean absolute deviations are given in Figure 3.5, and the MSE and RMSE of the deviations from true volatilities and correlations are given in Table 3.5.

Figure 3.5 Kernel density estimates of the deviations and absolute deviations of MCL and QML volatility and correlation estimates from the true ones, for the TVCMSV model. 

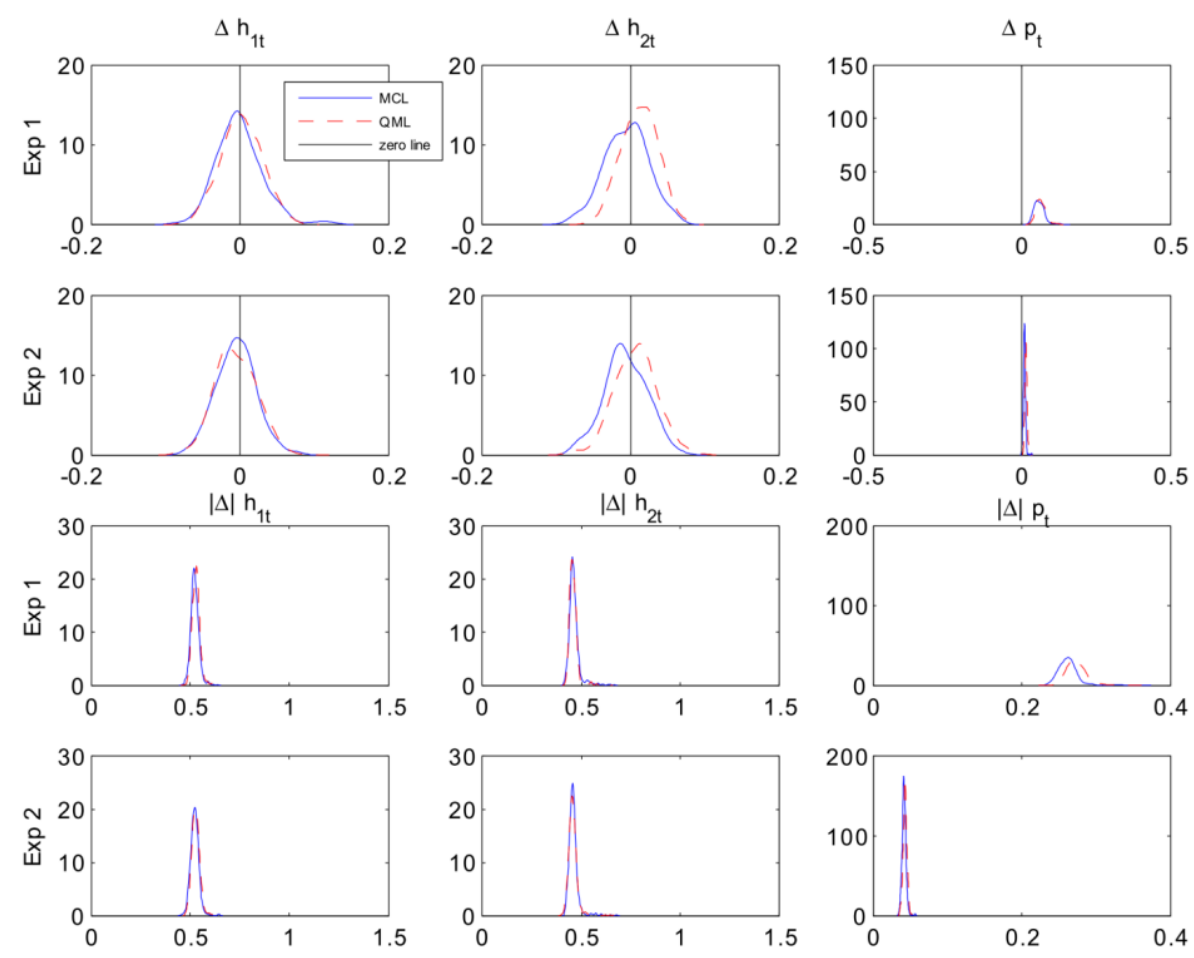

\begin{tabular}{lllll|llccc}
\hline \hline MAE & Method & $|\Delta| h_{1 t}$ & $|\Delta| h_{2 t}$ & $|\Delta| p_{t}$ & RMSE & Method & $\Delta h_{1 t}$ & $\Delta h_{2 t}$ & $\Delta p_{t}$ \\
\hline Exp1 & QML & 0.5286 & 0.4617 & 0.1025 & Exp1 & QML & 0.6672 & 0.5830 & 0.1307 \\
& MCL & 0.5226 & 0.4629 & 0.0998 & & MCL & 0.6569 & 0.5830 & 0.1265 \\
& QML/MCL & 1.0115 & 0.9974 & 1.0271 & & QML/MCL & 1.0156 & 0.9999 & 1.0334 \\
Exp2 & QML & 0.5270 & 0.4580 & 0.0364 & Exp2 & QML & 0.6670 & 0.5784 & 0.0567 \\
& MCL & 0.5214 & 0.4593 & 0.0355 & & MCL & 0.6554 & 0.5782 & 0.0542 \\
& QML/MCL & 1.0106 & 0.9972 & 1.0249 & & QML/MCL & 1.0177 & 1.0003 & 1.0456 \\
\hline \hline
\end{tabular}

Table 3.5 Mean absolute error (MAE) and root mean squared error (RMSE) of the QML and MCL volatility and correlation estimates for the TVCMSV model.

The QML and MCL parameter estimation results (except the correlation parameter) for the TVCMSV model are similar to those in the first experiment of the CCMSV model. For both experiments, the QML/MCL ratio of the deviations from true volatilities is less in the TVCMSV model than in the CCMSV model, mainly because MAE's and RMSE's of the deviations of MCL estimates of the volatilities from true ones are higher. Moreover the QML/MCL ratio of the MAE and RMSE of the deviations from true correlations is closer to 1 compared to the first experiment of CCMSV model. If we compare the two experiments of TVCMSV model, increasing the correlation did not change much the parameter estimation results or the QML/MCL ratio of the MAE and RMSE of the deviations from true volatilities and correlations. However, the MAE and RMSE of the deviations from true correlations decreased for both QML and MCL estimates. 


\begin{tabular}{l|cccccccccc}
\hline \hline Estim.|Param. & $\left\{P_{\varepsilon}\right\}_{21}$ & $\Gamma_{11}$ & $\Gamma_{21}$ & $\Phi_{11}$ & $\Phi_{22}$ & $L_{11}$ & $L_{22}$ & $\left\{Q_{\eta}\right\}_{11}$ & $\left\{Q_{\eta}\right\}_{21}$ & $\left\{Q_{\eta}\right\}_{22}$ \\
\hline Exp 1 - True & 0.2000 & -0.1000 & -0.1300 & 0.9000 & 0.9500 & -0.2000 & -0.2500 & 0.1500 & 0.0400 & 0.0800 \\
QML & 0.1278 & -0.1583 & -0.2376 & 0.8483 & 0.9085 & -0.1439 & -0.2131 & 0.2383 & 0.0525 & 0.1417 \\
& $(0.2429)$ & $(0.1710)$ & $(0.2737)$ & $(0.1474)$ & $(0.1056)$ & $(0.2363)$ & $(0.3514)$ & $(0.2794)$ & $(0.0653)$ & $(0.2157)$ \\
& {$[0.2534]$} & {$[0.1807]$} & {$[0.2940]$} & {$[0.1562]$} & {$[0.1135]$} & {$[0.2429]$} & {$[0.3533]$} & {$[0.2930]$} & {$[0.0665]$} & {$[0.2244]$} \\
MCL & 0.1995 & -0.1328 & -0.1700 & 0.8710 & 0.9351 & -0.1339 & -0.1932 & 0.1753 & 0.0447 & 0.0862 \\
& $(0.0458)$ & $(0.0735)$ & $(0.0883)$ & $(0.0645)$ & $(0.0328)$ & $(0.1395)$ & $(0.1970)$ & $(0.0838)$ & $(0.0275)$ & $(0.0371)$ \\
& {$[0.0458]$} & {$[0.0805]$} & {$[0.0969]$} & {$[0.0707]$} & {$[0.0360]$} & {$[0.1544]$} & {$[0.2050]$} & {$[0.0876]$} & {$[0.0279]$} & {$[0.0376]$} \\
\hline Exp 2 - True & 0.2000 & -0.1000 & -0.1300 & 0.9000 & 0.9500 & -0.5500 & -0.6000 & 0.1500 & 0.0400 & 0.0800 \\
QML & 0.1136 & -0.1705 & -0.2227 & 0.8342 & 0.9148 & -0.4002 & -0.4782 & 0.2443 & 0.0414 & 0.1122 \\
& $(0.2691)$ & $(0.1652)$ & $(0.2335)$ & $(0.1403)$ & $(0.0865)$ & $(0.2361)$ & $(0.3239)$ & $(0.2865)$ & $(0.0513)$ & $(0.1378)$ \\
& {$[0.2826]$} & {$[0.1796]$} & {$[0.2512]$} & {$[0.1549]$} & {$[0.0934]$} & {$[0.2796]$} & {$[0.3461]$} & {$[0.3016]$} & {$[0.0513]$} & {$[0.1415]$} \\
MCL & 0.2090 & -0.1624 & -0.1926 & 0.8477 & 0.9283 & -0.3161 & -0.4452 & 0.1999 & 0.0456 & 0.0902 \\
& $(0.0573)$ & $(0.1028)$ & $(0.0965)$ & $(0.0919)$ & $(0.0363)$ & $(0.1453)$ & $(0.1792)$ & $(0.1063)$ & $(0.0310)$ & $(0.0411)$ \\
& $(0.0580)$ & {$[0.1203]$} & {$[0.1151]$} & {$[0.1058]$} & {$[0.0424]$} & {$[0.2753]$} & $(0.2368)$ & $(0.1174)$ & $(0.0315)$ & $(0.0424)$ \\
\hline \hline
\end{tabular}

Table 3.6 The parameter estimation results of the simulations where the data is generated by an MSV model with diagonal leverage and estimated via QML and MCL methods.

Notes: For each experiment, the true parameter values are reported in the first row. Then for each estimation method, MC mean, standard deviation (in paranthesis) root mean squared error (in square brackets) of the parameter estimates are reported, respectively.

\begin{tabular}{llccc|llccc}
\hline \hline MAE & Method & $|\Delta| h_{1 t}$ & $|\Delta| h_{2 t}$ & $|\Delta| p$ & RMSE & Method & $\Delta h_{1 t}$ & $\Delta h_{2 t}$ & $\Delta p$ \\
\hline Exp1 & QML & 0.5437 & 0.4886 & 0.2112 & Exp1 & QML & 0.6870 & 0.6198 & 0.2534 \\
& MCL & 0.4502 & 0.3847 & 0.0363 & & MCL & 0.5684 & 0.4847 & 0.0458 \\
& QML/MCL & 1.2077 & 1.2701 & 5.8120 & & QML/MCL & 1.2087 & 1.2787 & 5.5343 \\
Exp2 & QML & 0.5860 & 0.5164 & 0.2311 & Exp2 & QML & 0.8763 & 0.6520 & 0.2826 \\
& MCL & 0.4600 & 0.3838 & 0.0399 & & MCL & 0.5853 & 0.4876 & 0.0580 \\
& QML/MCL & 1.2739 & 1.3455 & 5.7905 & & QML/MCL & 1.4973 & 1.3370 & 4.8741 \\
\hline \hline
\end{tabular}

Table 3.7 Mean absolute error (MAE) and root mean squared error (RMSE) of the QML and MCL volatility and correlation estimates for the MSV model with diagonal leverage.

For the MSV with diagonal leverage model we also perform two experiments, where we take all the parameter values from experiment 1 of the CCMSV model. For the additional parameters that control for the leverage we chose $L=\operatorname{diag}\{-0.2000,-0.2500\}$, which are similar to the values implied by the empirical estimates obtained in Asai and McAleer (2006) and $L=\operatorname{diag}\{-$ $0.5500,-0.6000\} .^{20}$ In Table 3.6, we report these true values of the parameters as well as the results of the QML and MCL estimations when the data was generated by an MSV model with diagonal leverage. The kernel density estimates of the mean and mean absolute deviations are given in Figure 3.6, and the MSE and RMSE of the deviations from true volatilities and correlations are given in Table 3.7. Compared with the first experiment of the CCMSV model, we observe that the performance of QML estimator of the correlation parameter decrease when leverage is introduced, while the performance of MCL estimator of the correlation parameter seems to remain similar. This result can be visually confirmed from the third column of Figure 3.6. In practice this means that for a given data, the QML estimate of the correlation parameter could possibly have a value that is very far from the true value. The RMSE's of the other parameter estimates are similar to those in the first experiment of the CCMSV model. When the leverage is low, the performance of the MCL estimator of the correlation parameter is similar to the case of CCMSV, but relatively worse when the leverage is high. The MAE and RMSE of the deviations of QML volatility estimates from true ones increase.

When we compare the two experiments with MSV with the diagonal leverage model, a higher leverage increased the MAE and RMSE of the deviations of QML volatility and correlation estimates from true ones. The QML/MCL ratios of these MAE's and RMSE's increased for the volatilities, but for correlations these ratios decreased since the MAE and RMSE of the deviations from true correlations increased for the MCL method. The efficiency of MCL estimators of the parameters decrease as the leverage increases, which is not the case for all

\footnotetext{
${ }^{20}$ When $L=\operatorname{diag}\{-0.2000,-0.2500\}$, the diagonal elements in the leverage matrix calculated from $Q_{\eta}^{*} L P_{\varepsilon}^{* \prime}$ (see Appendix 6.2) is $\{-0.0775,-0.0686\}$ and two examples from Table 3.2 in Asai and McAleer (2006) for these parameter estimates are $\{-0.0588,-0.0631\}$ and $\{-0.0648,-0.0476\}$.
} 
QML estimators. In both experiments, the MCL estimator of the leverage parameters, although having less standard deviation, seems to be further from the true values compared to the QML estimator.

Figure 3.6 Kernel density estimates of the deviations and absolute deviations of MCL and QML volatility and correlation estimates from the true ones, for the MSV model with diagonal leverage.
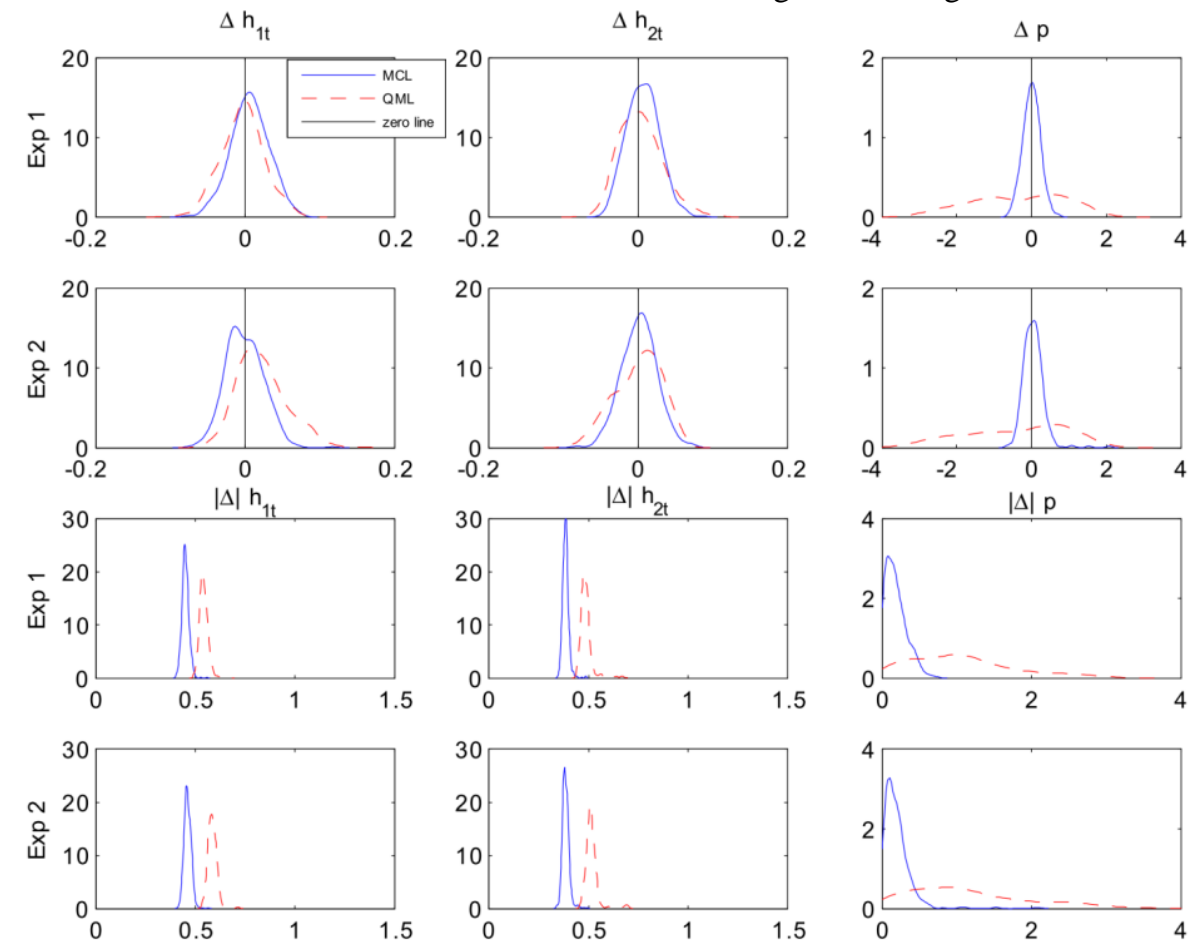

In two other MC experiments we consider the MSV model with non-diagonal leverage. The MCL estimation of this model requires the assumption that the leverage matrix is symmetric and positive or negative semi-definite. Therefore, we are interested in understanding what happens when this restriction is binding and not binding. In the first experiment (Exp 1) we consider a true leverage matrix that is symmetric but indefinite; therefore, the restriction of the MCL estimation is binding. In the second experiment $(\operatorname{Exp} 2)$, we consider a leverage matrix that is symmetric negative definite. The true values of the parameters, except the off-diagonal parameter of the leverage matrix, are taken from the first experiment of the MSV model with diagonal leverage. The off-diagonal parameter in the first experiment is randomly chosen to be high, while in the second experiment it is chosen to be as high as possible under the semidefiniteness restriction. The QML estimation does not require symmetricity or positive or negative semi-definiteness assumptions, although we assume that the leverage matrix is symmetric. Moreover, in the first experiment, we also estimate the same data with QML method and impose the restrictions on the leverage matrix, referring to the method as QML-restricted.

The parameter estimation results are given in Table 3.8 along with the true parameter values. The kernel density estimates of the mean and mean absolute deviations are given in Figure 3.7, and the MSE and RMSE of the deviations from true volatilities and correlations are given in Table 3.9. When we compare the first experiment with the first experiment of CCMSV we see that the unrestricted and restricted QML estimators of the correlation parameter are performing much worse, while the MCL estimator is performing similar. The MAE and RMSE of the deviations from true volatilities increased for all three estimators and they are very high for the unrestricted QML estimator compared to that of restricted QML and MCL. Compared to the first experiment of the CCMSV model, although QML-r/MCL ratios of the MAE's and RMSE's of the deviations from true volatilities are better, the relative performances of the correlation 
estimators of both QML methods are much worse than that of the MCL estimator. We obtained similar conclusions when comparing the second experiment with the first experiment of CCMSV model.

\begin{tabular}{l|cccccccccccc}
\hline \hline Estim.IParam. & $\left\{P_{\varepsilon}\right\}_{21}$ & $\Gamma_{11}$ & $\Gamma_{21}$ & $\Phi_{11}$ & $\Phi_{22}$ & $L_{11}$ & $L_{21}$ & $L_{22}$ & $\left\{Q_{\eta}\right\}_{11}$ & $\left\{Q_{\eta}\right\}_{21}$ & $\left\{Q_{\eta}\right\}_{22}$ \\
\hline Exp 1 - True & 0.2000 & -0.1000 & -0.1300 & 0.9000 & 0.9500 & -0.2000 & -0.2300 & -0.2500 & 0.1500 & 0.0400 & 0.0800 \\
QML & 0.1339 & -0.1676 & -0.2229 & 0.8402 & 0.9146 & -0.1752 & -0.1780 & -0.1927 & 0.2456 & 0.0436 & 0.1299 \\
- unrestricted & $(0.2498)$ & $(0.1637)$ & $(0.2268)$ & $(0.1364)$ & $(0.0854)$ & $(0.2516)$ & $(0.1787)$ & $(0.3310)$ & $(0.2685)$ & $(0.0570)$ & $(0.1829)$ \\
& {$[0.2584]$} & {$[0.1771]$} & {$[0.2450]$} & {$[0.1489]$} & {$[0.0924]$} & {$[0.2528]$} & {$[0.1861]$} & {$[0.3360]$} & {$[0.2850]$} & {$[0.0571]$} & {$[0.1896]$} \\
QML & 0.1291 & -0.1681 & -0.2266 & 0.8360 & 0.9133 & -0.1580 & -0.1442 & -0.2523 & 0.2622 & 0.0477 & 0.1356 \\
- restricted & $(0.2441)$ & $(0.1646)$ & $(0.2444)$ & $(0.1470)$ & $(0.0912)$ & $(0.2178)$ & $(0.1723)$ & $(0.3228)$ & $(0.2827)$ & $(0.0669)$ & $(0.1895)$ \\
& {$[0.2542]$} & {$[0.1782]$} & {$[0.2628]$} & {$[0.1604]$} & {$[0.0984]$} & {$[0.2219]$} & {$[0.1925]$} & {$[0.3229]$} & {$[0.3042]$} & {$[0.0673]$} & {$[0.1975]$} \\
MCL & 0.2016 & -0.1481 & -0.2091 & 0.8557 & 0.9208 & -0.1659 & -0.1232 & -0.2218 & 0.1890 & 0.0447 & 0.1033 \\
& $(0.0463)$ & $(0.0771)$ & $(0.1202)$ & $(0.0707)$ & $(0.0451)$ & $(0.1129)$ & $(0.0822)$ & $(0.1453)$ & $(0.0850)$ & $(0.0349)$ & $(0.0529)$ \\
& {$[0.0463]$} & {$[0.0908]$} & {$[0.1439]$} & {$[0.0834]$} & {$[0.0537]$} & {$[0.1180]$} & {$[0.1348]$} & {$[0.1480]$} & {$[0.0935]$} & {$[0.0352]$} & {$[0.0579]$} \\
\hline Exp 2 - True & 0.2000 & -0.1000 & -0.1300 & 0.9000 & 0.9500 & -0.2000 & -0.0500 & -0.2500 & 0.1500 & 0.0400 & 0.0800 \\
QML & 0.1317 & -0.1573 & -0.2544 & 0.8465 & 0.9017 & -0.1584 & -0.0326 & -0.1968 & 0.2339 & 0.0437 & 0.1421 \\
- unrestricted & $(0.2487)$ & $(0.1619)$ & $(0.3360)$ & $(0.1535)$ & $(0.1301)$ & $(0.2462)$ & $(0.1815)$ & $(0.3342)$ & $(0.2744)$ & $(0.0603)$ & $(0.1960)$ \\
& {$[0.2579]$} & {$[0.1717]$} & {$[0.3583]$} & {$[0.1625]$} & {$[0.1387]$} & {$[0.2497]$} & {$[0.1824]$} & {$[0.3384]$} & {$[0.2870]$} & {$[0.0604]$} & {$[0.2056]$} \\
MCL & 0.1999 & -0.1374 & -0.2029 & 0.8675 & 0.9226 & -0.1508 & -0.0104 & -0.2094 & 0.1777 & 0.0458 & 0.0994 \\
& $(0.0481)$ & $(0.0691)$ & $(0.1197)$ & $(0.0562)$ & $(0.0443)$ & $(0.1201)$ & $(0.1025)$ & $(0.1588)$ & $(0.0785)$ & $(0.0305)$ & $(0.0496)$ \\
& {$[0.0481]$} & {$[0.0785]$} & {$[0.1402]$} & {$[0.0649]$} & {$[0.0521]$} & {$[0.1297]$} & {$[0.1099]$} & {$[0.1639]$} & {$[0.0832]$} & {$[0.0311]$} & {$[0.0533]$} \\
\hline \hline
\end{tabular}

Table 3.8 The parameter estimation results of the simulations where the data is generated by an MSV model with non-diagonal leverage and estimated via unrestricted QML, restricted QML and MCL methods.

Notes: Experiment 1 refers to the case where the leverage matrix, $L$, is indefinite while in Experiment 2 it is (negative) definite. The restriction that was imposed to the QML estimation is the one that is required for MCL estimation, namely, the $L$ matrix is positive or negative semidefinite. For each experiment, the true parameter values are reported in the first row. Then for each estimation method, MC mean, standard deviation (in parenthesis) and root mean squared error (in square brackets) of the parameter estimates are reported, respectively.

\begin{tabular}{llccc|clccc}
\hline \hline MAE & Method & $|\Delta| h_{1 t}$ & $|\Delta| h_{2 t}$ & $|\Delta| p$ & RMSE & Method & $\Delta h_{1 t}$ & $\Delta h_{2 t}$ & $\Delta p$ \\
\hline Exp 1 & QML-u & 0.8156 & 0.8540 & 0.2175 & Exp 1 & QML-u & 1.0391 & 1.0970 & 0.2584 \\
& QML-r & 0.5539 & 0.5147 & 0.2088 & & QML-r & 0.6993 & 0.6550 & 0.2542 \\
& MCL & 0.5215 & 0.4532 & 0.0368 & & MCL & 0.6571 & 0.5723 & 0.0463 \\
& QML-u/MCL & 1.5641 & 1.8845 & 5.9094 & & QML-u/MCL & 1.5814 & 1.9170 & 5.5794 \\
& QML-r/MCL & 1.0621 & 1.1358 & 5.6728 & & QML-r/MCL & 1.0642 & 1.1445 & 5.4877 \\
Exp 2 & QML-u & 0.5427 & 0.4942 & 0.2181 & \multirow{2}{*}{ Exp 2 } & QML-u & 0.6852 & 0.6268 & 0.2579 \\
& MCL & 0.5149 & 0.4464 & 0.0368 & & MCL & 0.6485 & 0.5629 & 0.0481 \\
& QML-u/MCL & 1.0539 & 1.1070 & 5.9283 & & QML-u/MCL & 1.0566 & 1.1134 & 5.3635 \\
\hline \hline
\end{tabular}

Table 3.9 Mean absolute error (MAE) and root mean squared error (RMSE) of the QML and MCL volatility and correlation estimates for the MSV model with non-diagonal leverage.

Notes: In Experiment 1 the leverage matrix, $L$, is indefinite while in Experiment 2 it is (negative) definite.

In the first experiment comparing unrestricted and restricted QML estimation results, we see that the restricted QML estimate of the off-diagonal parameter of the leverage matrix is lower. This result confirms that the restriction was binding. Both unrestricted and restricted QML estimates of the correlation parameter are far from the true value. The MCL estimates have less RMSE than the unrestricted and restricted QML estimates for all the parameters, even though the MCL estimates of the off-diagonal element of the leverage matrix have high bias. When we look at the second experiment where the off-diagonal element of the leverage matrix was decreased (from Exp. 1 to Exp. 2), we observed less bias and RMSE for the MCL estimates of the parameters. As we observed in the first experiment, the QML estimate of the correlation parameter has a much higher RMSE than the MCL correlation estimate. This result can also be confirmed from the third column of Figure 3.7. 
Figure 3.7 Kernel density estimates of the deviations and absolute deviations of MCL and QML volatility and correlation estimates from the true ones, for the MSV model with non-diagonal leverage. Exp 1: true leverage matrix is indefinite. Exp 2: true leverage matrix is negative definite.
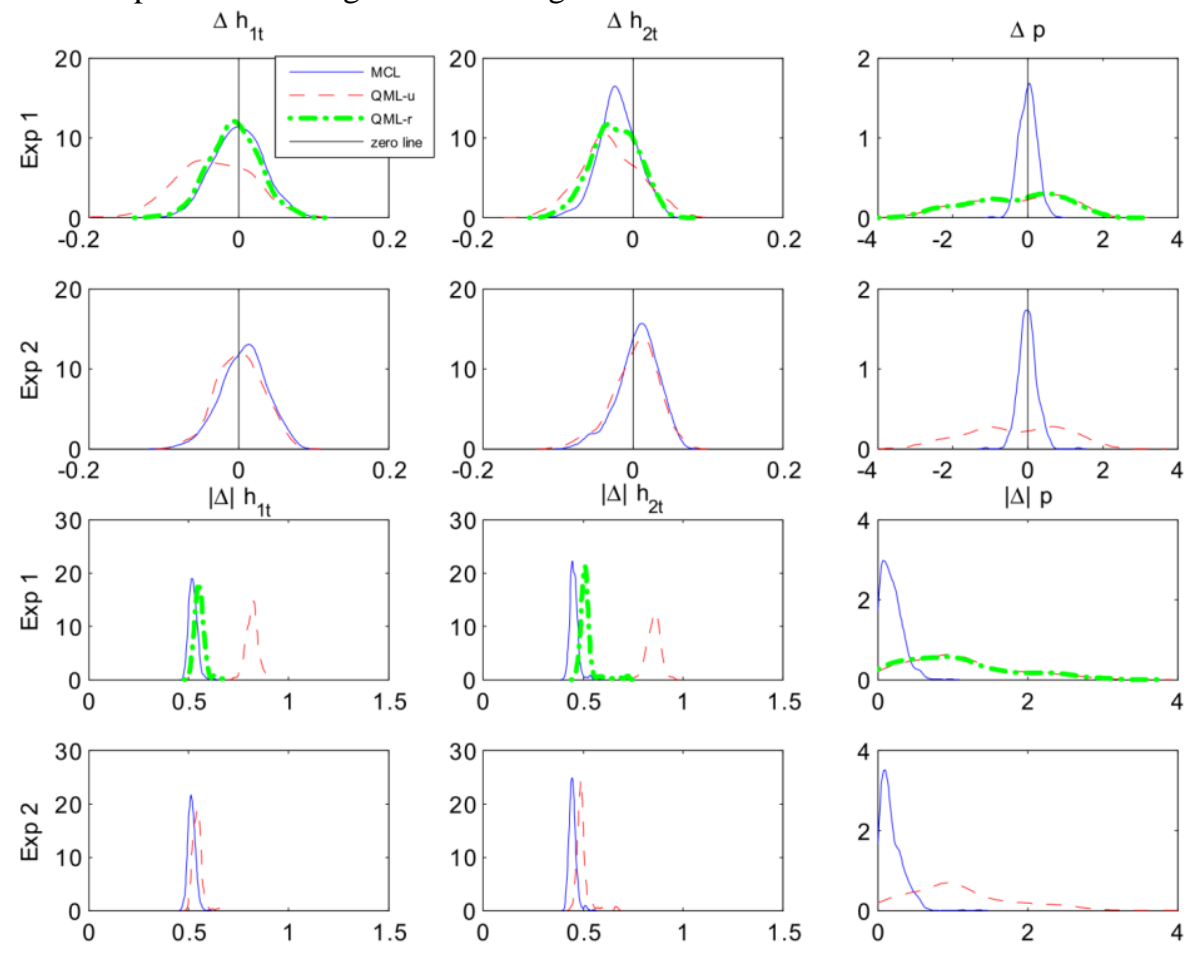

Overall, the restricted QML estimator seems to perform closer to the MCL estimator given that the same restriction is imposed. We also observed that the QML/MCL ratios of the MAE's and RMSE's of the deviations from true correlations increased from the first experiment to the second experiment. Although the restriction imposed in the MCL method could cause underestimation of the off-diagonal element of the leverage matrix as seen in Table 3.8, the volatilities and correlations are estimated by MCL with less RMSE compared to the corresponding unrestricted or restricted QML estimator. Finally, compared to the first experiment of the MSV model with diagonal leverage, the MAE and RMSE of the deviations from true volatilities are higher for the MCL method.

To sum up, our results confirm previous findings in the literature that the QML estimator is inefficient in terms of parameter estimation. In the case of the CCMSV model, we found that the performance of the QML estimator increases as the series become more correlated and also when the SV processes have higher variance. In all of the parameter sets considered, the performance of the MCL estimator was superior to that of the QML estimator. For the TVCMSV model, we took the parameter set where QML performed poorly in the case of the CCMSV model. The performance of the QML estimator relative to the MCL estimator was much better compared to the corresponding experiments of CCMSV model, even when the correlations were low. For the MSV models with leverage we also took the parameter set where QML performed poorly in the case of the CCMSV model. In both diagonal leverage and nondiagonal leverage cases, the QML performed worse than the corresponding case of the CCMSV model, while MCL performed similarly This result is actually intuitive because QML estimation is prone to the asymmetric distribution of the log-squared errors, and this asymmetry becomes more severe as the leverage increases. Hence, QML is performing poorly with MSV with leverage models, and more so when the leverage parameters are higher. It could be that the performance of the QML estimator improves with higher correlation in the data or higher variance of the SV processes, but we do not expect it to be better than the cases considered in the experiments with CCMSV model. For the nondiagonal leverage case, even when the 
restriction that MCL method requires that "the leverage matrix should be symmetric and positive or negative semi-definite" was binding, MCL was able to capture the underlying volatilities and correlations almost as well as in the corresponding case of the CCMSV model.

\section{AN EMPIRICAL EXAMPLE}

In this section our aim is to find empirical evidence supporting the use of the MSV model with non-diagonal leverage, i.e. the return shocks of one series is correlated with the volatility shocks of another series. For this estimation, a trivariate series of length 1717 is obtained from the daily returns of IBEX 35, FTSE 100, and DAX stock markets for the period between 4/1/2005 and 4/11/2011. ${ }^{21}$ The returns are calculated as: $100 \times \log \left(P_{t} / P_{t-1}\right)$. The descriptive statistics of the data is provided in Table 4.10. The IBEX 35 and DAX returns are skewed right, while FTSE100 is skewed left. On the other hand, as expected, all series depict high kurtosis. We also report the Box-Ljung statistics for serial correlation to 10 lags for the returns and its squared and logsquared transformations. Box-Ljung statistic for the return series, $y_{t}$, suggests that the data may not be random walks, more likely in the case of FTSE-100. On the other hand, there is strong evidence of nonlinearity in the squared returns and log-squared returns; suggesting that there is autocorrelation in these series.

\begin{tabular}{l|ccc}
\hline \hline Statistics $\backslash$ Series & IBEX-35 & FTSE-100 & DAX \\
\hline Mean & -0.0034 & 0.0076 & 0.0192 \\
SD & 1.5981 & 1.3667 & 1.5163 \\
Skewness & 0.1504 & -0.1385 & 0.0346 \\
Kurtosis & 10.7492 & 10.4146 & 9.7788 \\
Maximum & 13.4836 & 9.3842 & 10.7975 \\
Minimum & -9.5859 & -9.2646 & -7.4335 \\
\hline \multicolumn{2}{c}{ Box-Ljung test for autocorrelation, $p-$ values } \\
\hline Q(10), $y_{t}$ & $0.0179^{* *}$ & $0.0000^{* * *}$ & $0.0152^{* *}$ \\
Q(10), $y_{t}^{2}$ & $0.0000^{* * *}$ & $0.0000^{* * *}$ & $0.0000^{* * *}$ \\
Q(10), $\log y_{t}^{2}$ & $0.0000^{* * *}$ & $0.0000^{* * *}$ & $0.0000^{* * *}$ \\
\hline \hline
\end{tabular}

Table 4.10 Descriptive statistics of the returns.

Notes: *Significant at $10 \%,{ }^{* *}$ Significant at $5 \%,{ }^{* * *}$ Significant at $1 \%$.

A univariate SV model with leverage as in equations (2.7) and (2.8) is fit for each of the series. The QML and MCL (method of Jungbacker and Koopman, 2006) estimation results for the univariate model is given for each series in Table 4.11. From the results of the univariate estimation, we see that the MCL estimates imply more persistent SV processes compared to QML estimates. MCL estimates of the autoregressive parameter suggest that these SV processes are close to random walk. Also, MCL estimates of the leverage coefficients, the elements of $L$, are higher compared to QML estimates. Finally, the leverage parameter estimates are close to the parameters used in the second experiment of the MSV with diagonal leverage model.

The estimation of the MSV model with nondiagonal leverage requires the restriction that the $L$ matrix is symmetric and (positive or negative) semidefinite. This latter restriction is not required by the QML estimation. For comparison purposes however, we also estimated the model via QML assuming this restriction. The estimation results for the MSV model with nondiagonal leverage are given in Table 4.12. If we compare the results of the multivariate estimation with the results of the univariate estimation in Table 4.11, we see that the unconstrained and constrained QML estimates of the intercept, of the autoregressive parameter, and of the variance of the SV process are similar. On the other hand, the self-leverage of each series, that is the diagonal of the estimated $L$ matrix, is estimated to be less in magnitude for FTSE-100 and DAX indices compared to the univariate results.

\footnotetext{
${ }^{21}$ The data is taken from the finance.yahoo.com webpage at the date of estimation. We chose IBEX 35, FTSE 100 and DAX indices as they belong to the three of the largest stock markets in Europe.
} 


\begin{tabular}{l|l|ccccccc}
\hline \hline Estim. & Series & $\Gamma$ & $\Phi$ & $L$ & $Q_{\eta}$ & Log-like & AIC & BIC \\
\hline QML & IBEX 35 & 0.0137 & 0.9636 & -0.5262 & 0.0693 & -3890.6 & 7789.2 & 7811.0 \\
& & $(0.0012)$ & $(0.0055)$ & $(0.0944)$ & $(0.0080)$ & & & \\
& \multirow{2}{*}{ FTSE 100 } & 0.0003 & 0.9625 & -0.4964 & 0.0747 & -3911.7 & 7831.4 & 7853.1 \\
& & $(0.0038)$ & $(0.0057)$ & $(0.0457)$ & $(0.0066)$ & & & \\
& DAX & 0.0202 & 0.9480 & -0.6653 & 0.0801 & -3902.8 & 7813.5 & 7835.3 \\
& & $(0.0049)$ & $(0.0076)$ & $(0.0974)$ & $(0.0080)$ & & & \\
\hline MCL & IBEX 35 & 0.0025 & 0.9957 & -0.6574 & 0.0049 & -2456.7 & 4921.4 & 4943.2 \\
& & $(0.0002)$ & $(0.0012)$ & $(0.0007)$ & $(0.0002)$ & & & \\
& \multirow{2}{*}{ FTSE 100 } & -0.0001 & 0.9965 & -0.6022 & 0.0038 & -2149.1 & 4306.2 & 4328.0 \\
& & $(0.0001)$ & $(0.0014)$ & $(0.0312)$ & $(0.0015)$ & & & 4 \\
& \multirow{2}{*}{ DAX } & 0.0026 & 0.9942 & -0.8328 & 0.0044 & -2412.3 & 4832.5 & 4854.3 \\
& & $(0.0002)$ & $(0.0014)$ & $(0.0113)$ & $(0.0003)$ & & & \\
\hline \hline
\end{tabular}

Table 4.11 The empirical estimation results for the univariate SV model with leverage.

\begin{tabular}{|c|c|c|c|c|c|c|c|c|c|c|c|}
\hline $\begin{array}{l}\text { QML - unrestricted } \\
\text { Log-like: }-11179 \\
\text { AIC: } 22400 \\
\text { BIC: } 22515\end{array}$ & $\begin{array}{c}\left\{P_{\varepsilon}\right\}_{21} \\
0.8068 \\
(0.0232) \\
L_{31} \\
-0.0522 \\
(0.0024)\end{array}$ & $\begin{array}{c}\left\{P_{\varepsilon}\right\}_{31} \\
0.8720 \\
(0.0201) \\
L_{22} \\
-0.0851 \\
(0.0027)\end{array}$ & $\begin{array}{c}\left\{P_{\varepsilon}\right\}_{32} \\
0.8743 \\
(0.0150) \\
L_{32} \\
-0.1835 \\
(0.0110)\end{array}$ & $\begin{array}{c}\Gamma_{11} \\
0.0147 \\
(0.0015) \\
L_{33} \\
-0.2173 \\
(0.0124)\end{array}$ & $\begin{array}{c}\Gamma_{21} \\
0.0046 \\
(0.0014) \\
\left\{Q_{\eta}\right\}_{11} \\
0.0797 \\
(0.0069)\end{array}$ & $\begin{array}{c}\Gamma_{31} \\
0.0131 \\
(0.0017) \\
\left\{Q_{\eta}\right\}_{21} \\
0.0788 \\
(0.0034)\end{array}$ & $\begin{array}{c}\Phi_{11} \\
0.9612 \\
(0.0057) \\
\left\{Q_{\eta}\right\}_{31} \\
0.0718 \\
(0.0038)\end{array}$ & $\begin{array}{c}\Phi_{22} \\
0.9548 \\
(0.0065) \\
\left\{Q_{\eta}\right\}_{22} \\
0.0949 \\
(0.0058)\end{array}$ & $\begin{array}{c}\Phi_{33} \\
0.9581 \\
(0.0068) \\
\left\{Q_{\eta}\right\}_{32} \\
0.0754 \\
(0.0048)\end{array}$ & $\begin{array}{c}L_{11} \\
-0.5793 \\
(0.0284) \\
\left\{Q_{\eta}\right\}_{33} \\
0.0770 \\
(0.0081)\end{array}$ & $\begin{array}{c}L_{21} \\
-0.1573 \\
(0.0218)\end{array}$ \\
\hline $\begin{array}{l}\text { QML - restricted } \\
\text { Log-like: }-11181 \\
\text { AIC: } 22403 \\
\text { BIC: } 22518\end{array}$ & $\begin{array}{c}\left\{P_{\varepsilon}\right\}_{21} \\
0.8090 \\
(0.0226) \\
L_{31} \\
-0.0725 \\
(0.0143)\end{array}$ & $\begin{array}{c}\left\{P_{\varepsilon}\right\}_{31} \\
0.8730 \\
(0.0181) \\
L_{22} \\
-0.1245 \\
(0.0173)\end{array}$ & $\begin{array}{c}\left\{P_{\varepsilon}\right\}_{32} \\
0.8747 \\
(0.0161) \\
L_{32} \\
-0.1453 \\
(0.0299)\end{array}$ & $\begin{array}{c}\Gamma_{11} \\
0.0157 \\
(0.0010) \\
L_{33} \\
-0.2143 \\
(0.0177)\end{array}$ & $\begin{array}{c}\Gamma_{21} \\
0.0061 \\
(0.0028) \\
\left\{Q_{\eta}\right\}_{11} \\
0.0851 \\
(0.0041)\end{array}$ & $\begin{array}{c}\Gamma_{31} \\
0.0161 \\
(0.0024) \\
\left\{Q_{\eta}\right\}_{21} \\
0.0883 \\
(0.0036)\end{array}$ & $\begin{array}{c}\Phi_{11} \\
0.9609 \\
(0.0050) \\
\left\{Q_{\eta}\right\}_{31} \\
0.0839 \\
(0.0077)\end{array}$ & $\begin{array}{c}\Phi_{22} \\
0.9506 \\
(0.0071) \\
\left\{Q_{\eta}\right\}_{22} \\
0.1101 \\
(0.0080)\end{array}$ & $\begin{array}{c}\Phi_{33} \\
0.9522 \\
(0.0083) \\
\left\{Q_{\eta}\right\}_{32} \\
0.0899 \\
(0.0067)\end{array}$ & $\begin{array}{c}L_{11} \\
-0.6219 \\
(0.1125) \\
\left\{Q_{\eta}\right\}_{33} \\
0.0981 \\
(0.0168)\end{array}$ & $\begin{array}{c}L_{21} \\
-0.1738 \\
(0.0144)\end{array}$ \\
\hline $\begin{array}{l}\text { MCL } \\
\text { Log-like: }-4751 \\
\text { AIC: } 9543 \\
\text { BIC: } 9658 \\
\text { p_val/CCMSV: } 0.00\end{array}$ & $\begin{array}{c}\left.P_{\varepsilon}\right\}_{21} \\
0.8212 \\
(0.0001) \\
L_{31} \\
-0.1052 \\
(0.0019)\end{array}$ & $\begin{array}{c}\left\{P_{\varepsilon}\right\}_{31} \\
0.8297 \\
(0.0002) \\
L_{22} \\
-0.1769 \\
(0.0195)\end{array}$ & $\begin{array}{c}\left.P_{\varepsilon}\right\}_{32} \\
0.8542 \\
(0.0001) \\
L_{32} \\
-0.1387 \\
(0.0224)\end{array}$ & $\begin{array}{c}\Gamma_{11} \\
0.0070 \\
(0.0002) \\
L_{33} \\
-0.2657 \\
(0.0377)\end{array}$ & $\begin{array}{c}\Gamma_{22} \\
-0.0008 \\
(0.0001) \\
\left\{Q_{\eta}\right\}_{11} \\
0.0397 \\
(0.0001)\end{array}$ & $\begin{array}{c}\Gamma_{33} \\
0.0043 \\
(0.0003) \\
\left\{Q_{\eta}\right\}_{21} \\
0.0347 \\
(0.0001)\end{array}$ & $\begin{array}{c}\Phi_{11} \\
0.9751 \\
(0.0002) \\
\left\{Q_{\eta}\right\}_{31} \\
0.0373 \\
(0.0002)\end{array}$ & $\begin{array}{c}\Phi_{22} \\
0.9778 \\
(0.0002) \\
\left\{Q_{\eta}\right\}_{22} \\
0.0305 \\
(0.0001)\end{array}$ & $\begin{array}{c}\Phi_{33} \\
0.9755 \\
(0.0004) \\
\left\{Q_{\eta}\right\}_{32} \\
0.0327 \\
(0.0002)\end{array}$ & $\begin{array}{c}L_{11} \\
-0.6465 \\
(0.0001) \\
\left\{Q_{\eta}\right\}_{33} \\
0.0351 \\
(0.0001)\end{array}$ & $\begin{array}{c}L_{21} \\
-0.2151 \\
(0.0001)\end{array}$ \\
\hline $\begin{array}{l}\text { MCL-CCMSV } \\
\text { Log-like: }-5588 \\
\text { AIC: } 11207 \\
\text { BIC: } 11289\end{array}$ & $\begin{array}{c}\left\{P_{\varepsilon}\right\}_{21} \\
0.8413 \\
(0.0001) \\
\left\{Q_{\eta}\right\}_{31} \\
0.0331 \\
(0.0003)\end{array}$ & $\begin{array}{c}\left\{P_{\varepsilon}\right\}_{31} \\
0.8750 \\
(0.0002) \\
\left\{Q_{\eta}\right\}_{22} \\
0.0328 \\
(0.0002)\end{array}$ & $\begin{array}{c}\left\{P_{\varepsilon}\right\}_{32} \\
0.8835 \\
(0.0002) \\
\left\{Q_{\eta}\right\}_{32} \\
0.0298 \\
(0.0003)\end{array}$ & $\begin{array}{c}\Gamma_{11} \\
0.0053 \\
(0.0004) \\
\left\{Q_{\eta}\right\}_{33} \\
0.0327 \\
(0.0003)\end{array}$ & $\begin{array}{c}\Gamma_{22} \\
-0.0006 \\
(0.0002)\end{array}$ & $\begin{array}{c}\Gamma_{33} \\
0.0030 \\
(0.0003)\end{array}$ & $\begin{array}{c}\Phi_{11} \\
0.9812 \\
(0.0003)\end{array}$ & $\begin{array}{c}\Phi_{22} \\
0.9833 \\
(0.0002)\end{array}$ & $\begin{array}{c}\Phi_{33} \\
09827 \\
(0.0005)\end{array}$ & $\begin{array}{c}\left\{Q_{\eta}\right\}_{11} \\
0.0409 \\
(0.0002)\end{array}$ & $\begin{array}{c}\left\{Q_{\eta}\right\}_{21} \\
0.0339 \\
(0.0002)\end{array}$ \\
\hline $\begin{array}{l}\text { MCL-MSV Leverage } \\
\text { Log-like: }-10142 \\
\text { AIC: } 20319 \\
\text { BIC: } 20417 \\
\text { Model of Asai and } \\
\text { McAleer (2006) }\end{array}$ & $\begin{array}{c}\left\{P_{\varepsilon}\right\}_{21} \\
0.8188 \\
(0.0091) \\
\lambda_{3} \\
-0.0101 \\
(0.0210)\end{array}$ & $\begin{array}{c}\left\{P_{\varepsilon}\right\}_{31} \\
0.8363 \\
(0.0082) \\
\left\{Q_{\eta}\right\}_{11} \\
0.0589 \\
(0.0120)\end{array}$ & $\begin{array}{c}\left\{P_{\varepsilon}\right\}_{32} \\
0.8486 \\
(0.0069) \\
\left\{Q_{\eta}\right\}_{21} \\
0.0519 \\
(0.0098)\end{array}$ & $\begin{array}{c}\Gamma_{11} \\
-0.0011 \\
(0.0064) \\
\left\{Q_{\eta}\right\}_{31} \\
0.0445 \\
(0.0084)\end{array}$ & $\begin{array}{c}\Gamma_{22} \\
-0.0141 \\
(0.0061) \\
\left\{Q_{\eta}\right\}_{22} \\
0.0499 \\
(0.0103)\end{array}$ & $\begin{array}{c}\Gamma_{33} \\
-0.0087 \\
(0.0053) \\
\left\{Q_{\eta}\right\}_{32} \\
0.0438 \\
(0.0082)\end{array}$ & $\begin{array}{c}\Phi_{11} \\
0.9710 \\
(0.0063) \\
\left\{Q_{\eta}\right\}_{33} \\
0.0409 \\
(0.0090)\end{array}$ & $\begin{array}{c}\Phi_{22} \\
0.9728 \\
(0.0056)\end{array}$ & $\begin{array}{c}\Phi_{33} \\
0.9685 \\
(0.0064)\end{array}$ & $\begin{array}{c}\lambda_{1} \\
-0.0619 \\
(0.0236)\end{array}$ & $\begin{array}{c}\lambda_{2} \\
-0.0662 \\
(0.0185)\end{array}$ \\
\hline
\end{tabular}

Table 4.12 The empirical estimation results for the MSV with non-diagonal leverage model.

Notes: The data is obtained from the returns of IBEX 35, FTSE 100 and DAX stock markets (in order $1^{\text {st }}, 2^{\text {nd }}$ and $3^{r d}$ series). The estimation is performed via QML and MCL methods. Bollerslev-Wooldridge robust standard errors are obtained for the QML estimates while the standard errors of MCL estimates are obtained from the numerical approximation to the Hessian. Restricted QML estimation is the one where the restriction needed for MCL estimation is also employed in the QML estimation only for comparison reasons.

The MCL estimates of the autoregressive parameters are higher in the univariate estimation compared to the multivariate estimation, while the estimates of the self-leverage of each series are lower in the multivariate estimation. The MCL estimates of the autoregressive parameters are higher, whereas the MCL estimates of elements of the variance matrix of the SV process are lower; this is due to the fact that the estimation tries to match the unconditional variance in the data, and when the estimates of the autoregressive parameters are high, the variance matrix of the SV process is pushed downwards.

When we look at the leverage matrix estimates, we see that MCL estimates of the diagonal elements of $L$ matrix are higher compared to both of the QML estimates. The MCL leverage parameter estimates are statistically significant. Moreover, the likelihood ratio test to compare the MCL estimation results of CCMSV and MSV-NDL models suggest that the data is better explained by the latter model ${ }^{22}$. Figure 4.8 shows, for each series, the absolute values of the returns plotted along with the restricted QML and MCL smooth estimates of the standard

\footnotetext{
${ }^{22}$ The likelihood ratio test can't be used with the QML estimation because it is based on approximations.
} 
deviations. In the periods after the big volatility shocks, QML estimates of the underlying volatilities have been observed to be higher than the MCL estimates (for example around $t=1000$ ). Finally, the MCL estimates of the standard deviations follow the absolute values of the returns closely, while QML estimates are experiencing some jumps when volatility of the data is increasing.

Figure 4.8 Absolute values of the returns and the MCL and QML smooth estimates of the standard deviations for IBEX 35, FTSE 100 and DAX stock markets between 4/1/2005 - 4/11/2011. Data source: Yahoo Finance.
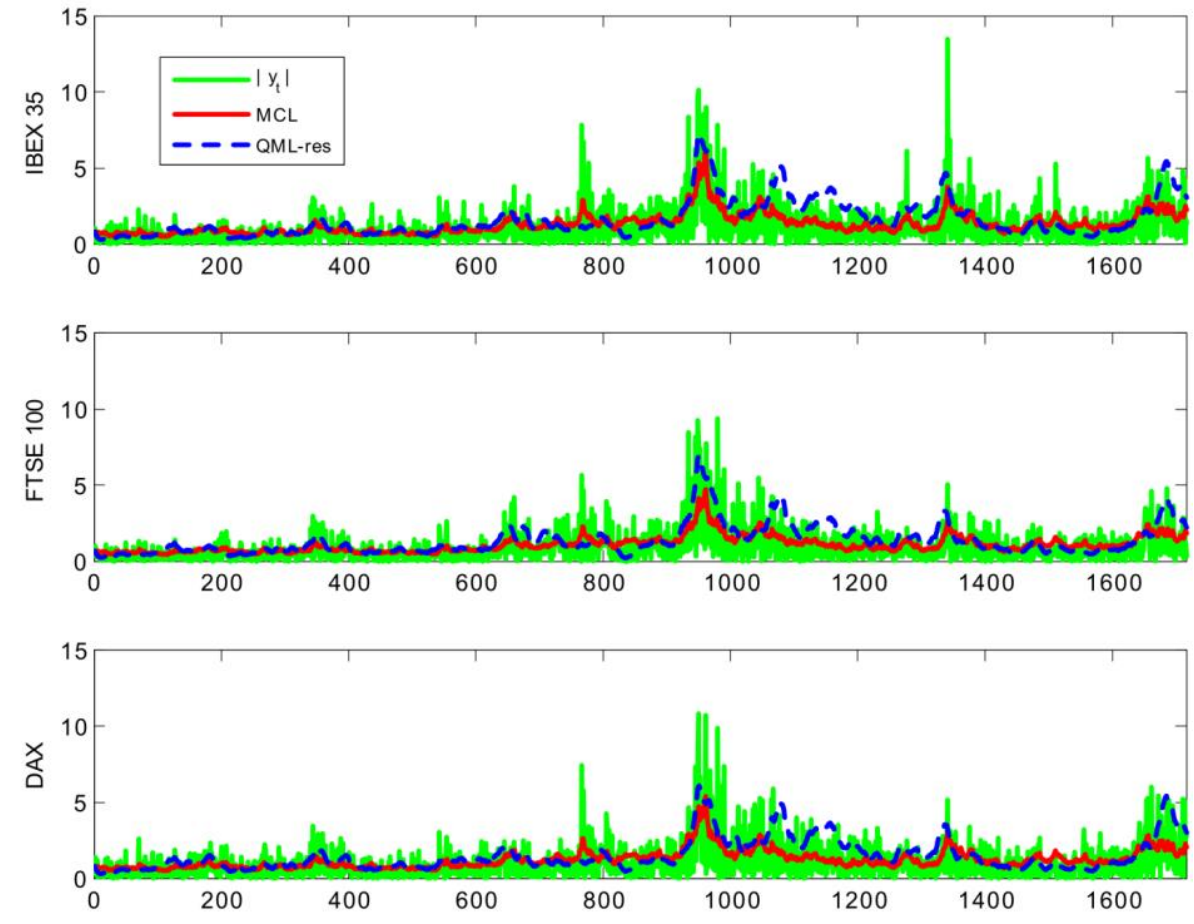

Another way to evaluate the results could be to see how much each model's estimate of volatilities are correlated true volatilities. To approximate the true volatilities, we use three different proxies: squared returns, rolling window estimate of volatilities with 60 days window, and Riskmetrics volatility estimates ${ }^{23}$. Table 4.13 and 4.14 give the correlations between the volatility proxies and volatility estimates in logarithms and levels, respectively. The first thing to notice is that correlations between levels are higher than they are between logs. In Table 4.13, the highest correlations are between the log-proxies and the log-volatility estimates of MSV with leverage model of Asai and McAleer (2006), with the exceptions of correlations between log-squared returns and log-volatility estimates of MSVNDL model for IBEX-35 and FTSE100 series. In Table 4.14, the highest correlations between the proxies and volatility estimates again correspond to MSV with leverage model of Asai and McAleer (2006), with the exceptions of the correlations between rolling window and Riskmetrics proxies and MSVNDL volatility estimates for FTSE-100.

We also make a comparison between the proxies and the volatility estimates based on some loss functions given below. If for series $i$ we would call our proxy of true volatility as $\sigma_{i t}^{2}$ and volatility estimate as $g_{i t}^{2}=\exp \left(h_{i t}\right)$ (where $h_{i t}$ is the log-volatility estimates):

\footnotetext{
${ }^{23}$ Andersen and Bollerslev (1998) show that $r_{t}^{2}$ is a very noisy estimate of the volatility.
} 


$$
\begin{array}{cc}
M S E_{2}=\frac{1}{T} \frac{1}{k} \sum_{i} \sum_{t}\left(\sigma_{i t}^{2}-g_{i t}^{2}\right)^{2} & R 2 L O G=\frac{1}{T} \frac{1}{k} \sum_{i} \sum_{t}\left(\log \left(\sigma_{i t}^{2} g_{i t}^{-2}\right)\right)^{2} \\
M S E_{1}=\frac{1}{T} \frac{1}{k} \sum_{i} \sum_{t}\left(\sigma_{i t}-g_{i t}\right)^{2} & M A D_{2}=\frac{1}{T} \frac{1}{k} \sum_{i} \sum_{t}\left|\left(\sigma_{i t}^{2}-g_{i t}^{2}\right)\right| \\
P S E=\frac{1}{T} \frac{1}{k} \sum_{i} \sum_{t}\left(\sigma_{i t}^{2}-g_{i t}^{2}\right)^{2} g_{i t}^{-4} & M A D_{1}=\frac{1}{T} \frac{1}{k} \sum_{i} \sum_{t}\left|\left(\sigma_{i t}-g_{i t}\right)\right| \\
Q L I K E=\frac{1}{T} \frac{1}{k} \sum_{i} \sum_{t}\left(\log \left(g_{i t}^{2}\right)-\sigma_{i t}^{2} g_{i t}^{-2}\right)^{2} &
\end{array}
$$

\begin{tabular}{|c|c|c|c|c|c|c|c|c|}
\hline IBEX-35 & $\begin{array}{l}\text { Log.Sq. } \\
\text { Ret. }\end{array}$ & $\begin{array}{l}\text { Log.Roll. } \\
\text { Wind. }\end{array}$ & $\begin{array}{c}\text { Log. } \\
\text { Riskmetrics }\end{array}$ & $\begin{array}{l}\text { MSVNDL- } \\
\text { QML_u }\end{array}$ & $\begin{array}{l}\text { MSVNDL- } \\
\text { QML_r }\end{array}$ & $\begin{array}{l}\text { MSVNDL- } \\
\text { MCL }\end{array}$ & $\begin{array}{l}\text { CCMSV- } \\
\text { MCL }\end{array}$ & MSVL_MCL \\
\hline Log.Sq.Ret. & 1.0000 & & & & & & & \\
\hline Log.Roll.Wind. & 0.3629 & 1.0000 & & & & & & \\
\hline Log.Riskmetrics & 0.4198 & 0.9346 & 1.0000 & & & & & \\
\hline MSVNDL-QML_u & 0.4218 & 0.7301 & 0.7436 & 1.0000 & & & & \\
\hline MSVNDL-QML_r & 0.4156 & 0.7355 & $0 . .7420$ & 0.9977 & 1.0000 & & & \\
\hline MSVNDL-MCL ${ }^{-}$ & 0.4511 & 0.7972 & 0.8542 & 0.8549 & 0.8428 & 1.0000 & & \\
\hline CCMSV-MCL & 0.4225 & 0.8177 & 0.8793 & 0.7188 & 0.7150 & 0.9200 & 1.0000 & \\
\hline MSVL_MCL & 0.4394 & 0.8361 & 0.8949 & 0.7825 & 0.7749 & 0.9402 & 0.9483 & 1.0000 \\
\hline FTSE-100 & $\begin{array}{l}\text { Log.Sq. } \\
\text { Ret. }\end{array}$ & $\begin{array}{l}\text { Log.Roll. } \\
\text { Wind. }\end{array}$ & $\begin{array}{c}\text { Log. } \\
\text { Riskmetrics }\end{array}$ & $\begin{array}{l}\text { MSVNDL- } \\
\text { QML_u }\end{array}$ & $\begin{array}{l}\text { MSVNDL- } \\
\text { QML_r }\end{array}$ & $\begin{array}{l}\text { MSVNDL- } \\
\text { MCL }\end{array}$ & $\begin{array}{c}\text { CCMSV- } \\
\text { MCL }\end{array}$ & MSVL_MCL \\
\hline Log.Sq.Ret. & 1.0000 & & & & & & & \\
\hline Log.Roll.Wind. & 0.3193 & 1.0000 & & & & & & \\
\hline Log.Riskmetrics & 0.3800 & 0.9426 & 1.0000 & & & & & \\
\hline MSVNDL-QML_u & 0.4195 & 0.6960 & 0.7190 & 1.0000 & & & & \\
\hline MSVNDL-QML_r & 0.4134 & 0.6881 & 0.7039 & 0.9973 & 1.0000 & & & \\
\hline MSVNDL-MCL & 0.4264 & 0.8019 & 0.8489 & 0.8500 & 0.8410 & 1.0000 & & \\
\hline CCMSV-MCL & 0.4026 & 0.8179 & 0.8702 & 0.7292 & 0.7145 & 0.9297 & 1.0000 & \\
\hline MSVL_MCL & 0.4174 & 0.8288 & 0.8831 & 0.7767 & 0.7620 & 0.9369 & 0.9492 & \\
\hline DAX & $\begin{array}{l}\text { Log.Sq. } \\
\text { Ret. }\end{array}$ & $\begin{array}{l}\text { Log.Roll. } \\
\text { Wind. }\end{array}$ & $\begin{array}{c}\text { Log. } \\
\text { Riskmetrics }\end{array}$ & $\begin{array}{l}\text { MSVNDL- } \\
\text { QML_u }\end{array}$ & $\begin{array}{l}\text { MSVNDL- } \\
\text { QML_r }\end{array}$ & $\begin{array}{l}\text { MSVNDL- } \\
\text { MCL }\end{array}$ & $\begin{array}{c}\text { CCMSV- } \\
\text { MCL }\end{array}$ & MSVL_MCL \\
\hline Log.Sq.Ret. & 1.0000 & & & & & & & NOS \\
\hline Log.Roll.Wind. & 0.3286 & 1.0000 & & & & & & \\
\hline Log.Riskmetrics & 0.3889 & 0.9330 & 1.0000 & & & & & \\
\hline MSVNDL-QML_u & 0.3968 & 0.7587 & 0.7772 & 1.0000 & & & & \\
\hline MSVNDL-QML_r & 0.3931 & 0.7433 & 0.7552 & 0.9968 & 1.0000 & & & \\
\hline MSVNDL-MCL & 0.3937 & 0.7478 & 0.8117 & 0.8109 & 0.8059 & 1.0000 & & \\
\hline CCMSV-MCL & 0.3822 & 0.7851 & 0.8558 & 0.7745 & 0.7577 & 0.9143 & 1.0000 & \\
\hline MSVL_MCL & 0.3952 & 0.7973 & 0.8711 & 0.8053 & 0.7874 & 0.9327 & 0.9504 & 1.0000 \\
\hline
\end{tabular}

Table 4.13 Correlations between logarithms of volatility proxies (e.g. log-squared returns) and log-volatility estimates.

These loss functions are used in Hansen and Lunde (2005). $M S E_{2}$ and $R 2 L O G$ are equivalent to using $R^{2}$ from the regressions of the true volatility and true log-volatility, respectively, on the volatility estimates. $M A D_{2}$ and $M A D_{1}$ are more robust to outliers than the other loss functions. $P S E$ measures the percentage squared errors and QLIKE is the loss function implied by a loglikelihood. On Table 4.15, we present the values of the loss functions for the three models and five estimators compared with three proxies. According to these results the losses are smaller whether for CCMSV or MSV with leverage model of Asai and McAleer (2006).

One reason why the MSV with leverage model of Asai and McAleer (2006) performed better than the MSV with nondiagonal leverage model could be that the true leverage matrix is indefinite, and the restriction that we impose to estimate the latter model is limiting its performance in estimating the volatility. A result supporting this possibility is that the unrestricted QML estimate of the leverage matrix is indefinite. 


\begin{tabular}{|c|c|c|c|c|c|c|c|c|}
\hline & Sq. & Roll. & & MSVNDL- & MSVNDL- & MSVNDL- & CCMSV- & \\
\hline IBEX-35 & Ret. & Wind. & Riskmetrics & QML_u & QML_r & MCL & MCL & MSVL_MCL \\
\hline Sq.ret. & 1.0000 & & & & & & & \\
\hline Roll.wind. & 0.2923 & 1.0000 & & & & & & \\
\hline Riskmetrics & 0.4705 & 0.8588 & 1.0000 & & & & & \\
\hline MSVNDL-QML_u & 0.4168 & 0.5928 & 0.7625 & 1.0000 & & & & \\
\hline MSVNDL-QML_r & 0.4091 & 0.5881 & 0.7562 & 0.9964 & 1.0000 & & & \\
\hline MSVNDL-MCL & 0.5092 & 0.5938 & 0.8352 & 0.8421 & 0.8250 & 1.0000 & & \\
\hline CCMSV-MCL & 0.5074 & 0.5554 & 0.8159 & 0.7417 & 0.7332 & 0.9364 & 1.0000 & \\
\hline MSVL_MCL & 0.5270 & 0.6044 & 0.8562 & 0.8261 & 0.8116 & 0.9722 & 0.9445 & 1.0000 \\
\hline & & Roll. & & MSVNDL- & MSVNDL- & MSVNDL- & CCMSV- & \\
\hline FTSE-100 & Sq.ret. & Wind. & Riskmetrics & QML_u & QML_r & MCL & MCL & MSVL_MCL \\
\hline $\begin{array}{l}\text { Sq.ret. } \\
\text { Roll.wind. }\end{array}$ & $\begin{array}{l}1.0000 \\
0.3602\end{array}$ & 1.0000 & & & & & & \\
\hline Riskmetrics & 0.5316 & 0.8841 & 1.0000 & & & & & \\
\hline MSVNDL-QML_u & 0.5146 & 0.5928 & 0.8171 & 1.0000 & & & & \\
\hline MSVNDL-QML_r & 0.5068 & 0.5769 & 0.8026 & 0.9982 & 1.0000 & & & \\
\hline MSVNDL-MCL & 0.5470 & 0.6381 & 0.8708 & 0.8856 & 0.8716 & 1.0000 & & \\
\hline CCMSV-MCL & 0.5413 & 0.5887 & 0.8463 & 0.8593 & 0.8474 & 0.9558 & 1.0000 & \\
\hline MSVL_MCL & 0.5610 & 0.6252 & 0.8666 & 0.8863 & 0.8737 & 0.9670 & 0.9742 & 1.0000 \\
\hline & & Roll. & & MSVNDL- & MSVNDL- & MSVNDL- & CCMSV- & \\
\hline $\begin{array}{l}\text { DAX } \\
\text { Sq.ret. }\end{array}$ & $\begin{array}{l}\text { Sq.Ret. } \\
1.0000\end{array}$ & Wind. & Riskmetrics & QML_u & QML_r & MCL & MCL & MSVL_MCL \\
\hline Roll.wind. & 0.3388 & 1.0000 & & & & & & \\
\hline Riskmetrics & 0.5019 & 0.8830 & 1.0000 & & & & & \\
\hline MSVNDL-QML_u & 0.4306 & 0.6163 & 0.7635 & 1.0000 & & & & \\
\hline MSVNDL-QML_r & 0.4226 & 0.5821 & 0.7355 & 0.9960 & 1.0000 & & & \\
\hline MSVNDL-MCL & 0.5350 & 0.5405 & 0.7924 & 0.7686 & 0.7551 & 1.0000 & & \\
\hline CCMSV-MCL & 0.5064 & 0.5699 & 0.8071 & 0.7614 & 0.7458 & 0.9541 & 1.0000 & \\
\hline MSVL_MCL & 0.5416 & 0.6146 & 0.8567 & 0.8072 & 0.7915 & 0.9610 & 0.9663 & 1.0000 \\
\hline
\end{tabular}

Table 4.14 Correlations between volatility proxies (e.g. squared returns) and volatility estimates.

\section{CONCLUSIONS}

In this paper we compare the performance of Quasi Maximum Likelihood estimation method of Harvey et al. (1994) and Monte Carlo Likelihood estimation method of Jungbacker and Koopman (2006) in estimating the parameters, as well as in estimating the underlying volatilities and correlations via Monte Carlo experiments. For this comparison, we consider the Constant Correlation MSV (CCMSV) model of Harvey et al. (1994), the Time Varying Correlation MSV (TVCMSV) model of Jungbacker and Koopman (2006), and two MSV models with leverage that we discussed in the text. We also provide the transformations of the MSV models with leverage necessary for the estimation.

According to our results MCL has better finite sample performance than QML. This finding is in accordance with the existing results in the literature. On the other hand, we conclude that (1) when the correlations are high and/or dynamic, (2) when the SV variances are high, (3) when the model doesn't include leverage effects, QML method can be used. Given the results in the literature on the inefficiency of QML estimator in small samples, having a larger sample size when using QML method would be an important plus. In other cases, the MCL method should be preferred. The implementation of MCL method is relatively more complicated than the QML estimation and requires much more time to converge. Moreover, the analytical derivatives needed for the MCL estimation may be harder to obtain with large cross-sections. One could choose to use numerical derivatives, but the derivatives obtained by numerical approximation for large state vectors could be time consuming and numerically unstable. Hence, having an idea beforehand if QML estimator will be fine to use is an advantage. A final note is that while the MCL method can be considered for fitting the returns of few international stock markets, the QML method could be used for the estimation of models with larger cross sections.

As for further research, the inefficiency of the QML method could be improved partially by employing a nonlinear filter instead of the Kalman filter. The latter is a linear filter and therefore 
leads to minimum mean square linear estimators rather than minimum mean squared estimators. Watanabe (1999) provides a nonlinear filter for QML estimation for the univariate SV model, and extending it to a multivariate setup would be an interesting topic for further study.

Another point to consider would be to introduce a correlation between the SV process errors and the stochastic correlation parameter errors in the Tsay (2005) model. The intuition behind this extra parameter would be that the volatility shocks are correlated with the correlation shocks. As commonly observed in crisis periods, the markets tend to react similarly when there are bad news. The extra parameter can capture this fact by suggesting that when there is a shock that increases volatility, there is a shock that increases correlation.

\section{APPENDIX}

\section{A.1 Derivatives}

Following Jungbacker and Koopman (2006) and Lutkepohl (1996), we obtained the derivatives for the bivariate MSV model with diagonal leverage needed for deriving the approximating linear model. For the nondiagonal leverage model, it can be easily modified. On the other hand, these derivatives are extendable to cases with more than $k=2$ series; in the empirical estimation part these derivatives are used for $k=3$ case.

$$
y_{t}=H_{t}^{1 / 2} P_{\varepsilon}^{*} \varepsilon_{t} \Rightarrow \varepsilon_{t}=\left(P_{\varepsilon}^{*}\right)^{-1} H_{t}^{-1 / 2} y_{t}
$$

$H_{t}$ and $P_{\varepsilon}^{*}$ as defined in (2.1) and (2.3). Then using (2.9) we can write:

$$
d_{t}=\left(P_{\varepsilon}^{*}\right)^{-1} H_{t}^{-1 / 2} y_{t}-S\left(Q_{\eta}^{*}\right)^{-1} \alpha_{2, t}
$$

$\alpha$ defined as in (2.11). If we let $X=I_{2}-S L O$ where $I_{2}$ is a $2 \times 2$ identity matrix and $\alpha_{1, t}$ be the volatilities part of $\alpha_{t}$ then the loglikelihood for (2.10) would be given by:

$$
\log p\left(y_{t} \mid h_{t}\right)=-0.5 k \log (2 \pi)-0.5 \sum_{i} \alpha_{1, t, i}-0.5 \log \left(\operatorname{det}\left(P_{\varepsilon}^{*} X\left(P_{\varepsilon}^{*}\right)^{\prime}\right)\right)-0.5 d_{t}^{\prime} X^{-1} d_{t}
$$

Then the first derivatives with respect to the state vector $\alpha_{t}$ would be given by:

$$
\begin{gathered}
\frac{\partial l_{t}}{\partial \alpha_{t}}=-0.5\left[\begin{array}{l}
1 \\
1 \\
0 \\
0
\end{array}\right]-0.5 \frac{\partial d_{t}^{\prime}}{\partial \alpha_{t}}\left(X^{-1}+\left(X^{-1}\right)^{\prime}\right) d_{t} \\
\frac{\partial d_{t}^{\prime}}{\partial \alpha_{t}}=\left(\frac{\partial d_{t}}{\partial \alpha_{t}}\right)^{\prime}=-\left(\begin{array}{c}
\left\{-0.5\left(P_{\varepsilon}^{*}\right)^{-1} \operatorname{diag}\left(H_{t}^{-1 / 2} y_{t}\right)\right\}^{\prime} \\
-\left\{S\left(Q_{\eta}^{*}\right)^{-1}\right\}^{\prime}
\end{array}\right)
\end{gathered}
$$

The second derivatives are obtained from:

$$
\frac{\partial^{2} l_{t}}{\partial \alpha_{t} \partial \alpha_{t}^{\prime}}=-0.5\left\{\frac{\partial d_{t}^{\prime}}{\partial \alpha_{t}}\left(X^{-1}+\left(X^{-1}\right)^{\prime}\right) \frac{\partial d_{t}}{\partial \alpha_{t}^{\prime}}+\left[d_{t}^{\prime}\left(X^{-1}+\left(X^{-1}\right)^{\prime}\right) \otimes I_{4}\right] \frac{\partial \operatorname{vec}\left(\frac{\partial d_{t}^{\prime}}{\partial \alpha_{t}}\right)}{\partial \alpha_{t}^{\prime}}\right\}
$$

where $I_{4}$ is a $4 \times 4$ identity matrix and $\otimes$ is a Kronecker product. The last expression in the equation is equal to: 


$$
\frac{\partial \operatorname{vec}\left(\frac{\partial d_{t}^{\prime}}{\partial \alpha_{t}}\right)}{\partial \alpha_{t}^{\prime}}=0.25 Z
$$

where $Z_{1,1}=\left\{\left(P_{\varepsilon}^{*}\right)^{-1} \operatorname{diag}\left(H_{t}^{1 / 2} y_{t}\right)\right\}_{1,1}, Z_{5,1}=\left\{\left(P_{\varepsilon}^{*}\right)^{-1} \operatorname{diag}\left(H_{t}^{-1 / 2} y_{t}\right)\right\}_{2,1}$, and $Z_{6,2}=\left\{\left(P_{\varepsilon}^{*}\right)^{-1} \operatorname{diag}\left(H_{\bar{t}}^{1 / 2} y_{t}\right)\right\}_{2,2}$, while the rest of the entries are zeros.

\section{A.2 Relation of MSV Models with Leverage to the MSV_L model of Asai and McAleer (2006)}

Following the notation used in equations (2.7) and (2.8), we can write the model of Asai and McAleer (2006) as follows:

$$
\begin{gathered}
y_{t}=H_{t}^{1 / 2} \varepsilon_{t} \\
h_{t+1}=\Gamma+\Phi h_{t}+\eta_{t}
\end{gathered}
$$

with the following distribution of the errors:

$$
\left(\begin{array}{c}
\varepsilon_{t} \\
\eta_{t}
\end{array}\right) \sim N\left(0_{2 k},\left[\begin{array}{cc}
P_{\varepsilon} & \bar{L} \\
\bar{L} & Q_{\eta}
\end{array}\right]\right)
$$

where $P_{\varepsilon}$ is the correlation matrix of $\varepsilon_{t}, \bar{L}=\operatorname{diag}\left(\lambda_{1} \sigma_{11}^{1 / 2}, \lambda_{2} \sigma_{22}^{1 / 2}, \ldots, \lambda_{k} \sigma_{k k}^{1 / 2}\right)$ and $Q_{\eta}$ is the covariance of $\eta_{t}$ such that $Q_{\eta}=\left\{\sigma_{\eta}, i j\right.$.

The relation between diagonal leverage matrix in Asai and McAleer (2006), $\bar{L}$, and the nondiagonal leverage matrix in our MSV with leverage models, $L$, can be deduced from equations (2.7) and (2.8) in the following manner:

$$
\operatorname{Var}\left(\left[\begin{array}{c}
P_{\varepsilon}^{*} \varepsilon_{t} \\
Q_{\eta}^{*} \eta_{t}
\end{array}\right]\right)=\left[\begin{array}{cc}
P_{\varepsilon}^{*} & 0_{k} \\
0_{k} & Q_{\eta}^{*}
\end{array}\right]\left[\begin{array}{cc}
I_{\varepsilon}^{*} & L \\
L & I
\end{array}\right]\left[\begin{array}{cc}
\left(P_{\varepsilon}^{*}\right)^{\prime} & 0_{k} \\
0_{k} & \left(Q_{\eta}^{*}\right)^{\prime}
\end{array}\right]=\left[\begin{array}{cc}
P_{\varepsilon} & P_{\varepsilon}^{*} L\left(Q_{\eta}^{*}\right)^{\prime} \\
Q_{\eta}^{*} L\left(P_{\varepsilon}^{*}\right)^{\prime} & Q_{\eta}
\end{array}\right]
$$

Notice that $Q_{\eta}^{*} L P_{\varepsilon}^{* \prime}$, and $P_{\varepsilon}^{*} L Q_{\eta}^{* \prime}$ are not diagonal. The assumption used in Asai and McAleer (2006) is that $Q_{\eta}^{*} L P_{\varepsilon}^{* \prime}$ is a diagonal matrix. Hence, as mentioned in the paper, both MSV models with diagonal and non-diagonal leverage matrices discussed in this paper offer richer structure on the leverage behaviour in the data. 


\section{REFERENCES}

Aguilar, O. and M. West (2000). Bayesian Dynamic Factor Models and Portfolio Allocation. Journal of Business and Economic Statistics, 18, 338-357.

Alizadeh, S., M.W. Brandt and F.X. Diebold (2002). Range based estimation of stochastic volatility models. Journal of Finance, 57, 1047-1091.

Andersen, T., H. Chung and B. Sorensen (1999). Efficient method of moments estimation of a stochastic volatility model: a Monte Carlo study. Journal of Econometrics, 91, 61-87.

Andersen, T. and T. Bollerslev (1998). Answering the skeptics: Yes, standard volatility models do provide accurate forecasts. International Economic Review, 39 (4), 885-905.

Asai, M. and M. McAleer (2005). Dynamic asymmetric leverage in stochastic volatility models. Econometric Reviews, 24, 317-332.

Asai, M. and M. McAleer (2006). Asymmetric Multivariate Stochastic Volatility. Econometric Reviews, 25 (2-3), 453-473.

Asai, M., M. McAleer and J. Yu (2006). Multivariate Stochastic Volatility: A Review. Econometric Reviews, 25 (2-3), 145-175.

Asai, M. and M. McAleer (2009). The structure of dynamic correlations in multivariate stochastic models. Journal of Econometrics, 150, 182-192.

Asai, M. and M. McAleer (2011). Dynamic conditional correlations for asymmetric processes. Journal of the Japan Statistical Society, 41 (2), 143-157.

Asai, M. and M. McAleer (2015). Leverage and feedback effects on multifactor Wishart stochastic volatility for option pricing. Journal of Econometrics, 187 (2), 436-446.

Asai, M. and A. Unite (2008). The relationship between stock return volatility and trading volume: the case of the Philippines. Applied Financial Economics, 18 (16), 1333-1341.

Bauwens, L., L. Sebastien and J.V.K. Rombouts (2006). Multivariate GARCH Models: A Survey. Journal of Applied Econometrics, 21, 79-109.

Black, F. (1976). Studies in Stock Price Volatility Changes. In Proceedings of the 1976 Business Meeting of the Business and Economic Section, American Economic Association, 177-181.

Bollerslev, T. (1986). Generalized autoregressive conditional heteroskedasticity. Journal of Econometrics, 31 (3), 307-327

Bollerslev, T., R.Y. Chou and K.F. Kroner (1992). ARCH modelling in finance: A review of the theory and empirical evidence. Journal of Econometrics, 52, 5-59

Bollerslev, T. and M.J. Wooldridge (1992). Quasi-maximum likelihood estimation and inference in dynamic models with time-varying covariances. Econometric Reviews, 11 (2), 143-172. 
Brandt, M.W. and Q. Kang (2004). On the relationship between the conditional mean and volatility of stocks returns: a latent VAR approach. Journal of Financial Economics, 72, 217-258.

Breidt, F.J. and A. Carriquiry (1996). Improved quasi-maximum likelihood estimation for stochastic volatility models. In: (Eds.) Modelling and Prediction: Honouring Seymour Geisel, ed. A. Zellner and J.S. Lee. New York: Springer.

Broto, C. and E. Ruiz (2004). Estimation methods for stochastic volatility models: a survey. Journal of Economic Surveys, 18 (5), 613-649.

Carnero, A., D. Pena and E. Ruiz (2004). Persistence and Kurtosis in GARCH and Stochastic Volatility Models. Journal of Financial Econometrics, 2 (2), 319-342.

Chib, S., F. Nardari and N. Shephard (2006). Analysis of High Dimensional Multivariate Stochastic Volatility Models. Journal of Econometrics, 134 (2), 341-371

Chib, S., Y. Omori and M. Asai (2009). Multivariate Stochastic Volatility. Handbook of Financial Time Series, Part 2, 365-400.

Christie, A. (1982). The Stochastic Behavior of Common Stock Variances- Value, Leverage, and Interest Rate Effects. Journal of Financial Economic Theory, 10, 407-432.

Danielsson, J. (1998). Multivariate Stochastic Volatility Models: Estimation and A Comparison With VGARCH models. Journal of Empirical Finance, 5, 155-173.

Danielsson, J. and J.F. Richard (1993). Accelerated Gaussian Importance Sampler with Application to Dynamic Latent Variable Models. Journal of Applied Econometrics, 8, $153-154$

Danielsson, J. (1994). Stochastic volatility in asset prices estimation with simulated maximum likelihood, Journal of Econometrics, 64, Issues 1-2, 375-400

De Jong, P. and N. Shephard (1995). The simulation smoother for time series models. Biometrika, 82 (2), 339-350.

Dunsmuir, W. (1979). A Central Limit Theorem for Parameter Estimation in Stationary Time Series and Its Applications to Models for a Signal Observed White Noise. Annals of Statistics, 7, 490-506.

Durbin, J. and S.J. Koopman (1997). Monte Carlo maximum likelihood estimation for nonGaussian state space models. Biometrika, 84 (3), 669-684.

Durbin, J. and S.J. Koopman (2002). A simple and efficient simulation smoother for state space time series analysis. Biometrika, 89, (3), 603-615

Engle, R.F. (1982). Autoregressive conditional heteroskedasticity with estimates of the variance of United Kingdom inflation. Econometrica, 50 (4), 987-1008.

Fuller, W.A. (1996). Introduction to Statistical Time Series. New York: Wiley. 
Ghysels, E., A.C. Harvey and E. Renault (1996). Stochastic volatility. In Statistical Models in Finance, ed. C.R. Rao and G.S. Maddala. Amsterdam: North-Holland, 119-191.

Hansen, P.R. and A. Lunde (2005). A forecast comparison of volatility models: does anything beat a GARCH(1,1)? Journal of Applied Econometrics, 20, 873-889.

Harvey, A. (1989). Forecasting Structural Models and the Kalman Filter. Cambridge University Press: Cambridge

Harvey, A., E. Ruiz and N. Shephard (1994). Multivariate stochastic variance models. The Review of Economic Studies, 61, 247-264.

Harvey, A.C. and N.G. Shephard (1996). Estimation of an asymmetric stochastic volatility model for asset returns. Journal of Business and Economic Statistics, 14, 429-434.

Hwang, S. and S.E. Satchell (2000). Market risk and the concept of fundamental volatility: measuring volatility across asset and derivative markets and testing for the impact of derivatives markets on financial markets. Journal of Banking and Finance, 24, 759-785.

Hull, J. and A. White (1987). Hedging the Risks from Writing Foreign Currency Options. Journal of International Money and Finance, 6, 131-152.

Jacquier, E., N. Polson and P. Rossi (1994). Bayesian Analisis of Stochastic Volatility Models, Journal of Business and Economic Statistics, 12, 371-389

Jungbacker, B. and S.J. Koopman (2005). On Importance Sampling for State Space Models, Tinbergen Institute Discussion Paper No. 05-117. Available at SSRN: http://ssrn.com/abstract=873472 (accessed June 17, 2016).

Jungbacker, B. and S.J. Koopman (2006). Monte Carlo likelihood estimation for three multivariate stochastic volatility models. Econometric Reviews, 25 (2-3), 385-408.

Kim, S., N. Shephard and S. Chib (1998). Stochastic Volatility: Likelihood Inference and Comparison with ARCH Models. Review of Economic Studies, 65 (3), 361-393.

Liesenfeld, R. (1998). Dynamic bivariate mixture models: modeling the behavior of prices and trading volume. Journal of Business and Economic Statistics, 16, 101-109.

Liesenfeld, R. (2001). A generalized bivariate mixture model for stock price volatility and trading volume. Journal of Econometrics, 104, 141-178.

Liesenfeld, R. and R.C. Jung (2000). Stochastic volatility models: conditional normality versus heavy tailed distributions. Journal of Applied Econometrics, 15, 137-160.

Liesenfeld, R. and J.F. Richard (2003). Univariate and Multivariate Stochastic Volatility Models: Estimation and Diagnostics. Journal of Empirical Finance, 10, 505-531.

Liesenfeld, R. and J.F. Richard (2006). Classical and Bayesian Analysis of Univariate and Multivariate Stochastic Volatility Models. Econometric Reviews, 25 (2-3), 335-360 
Lutkepohl, H. (1996). Handbook of Matrices. New York: Wiley.

Maasoumi, E. and M. McAleer (2006). Multivariate Stochastic Volatility: An Overview. Econometric Reviews, 25 (2-3), 139-144.

Meyer, R. and J. Yu (2000). BUGS for a bayesian analysis of stochastic volatility models. Econometrics Journal, 3, 198-215.

Nelson, D.B. (1988). Time Series Behavior of Stock Market Volatility and Returns. Unpublished PhD dissertation. Economics Dept.: MIT:

Omori, Y. and T. Ishihara (2012) Multivariate Stochastic Volatility Models, in Handbook of Volatility Models and Their Applications. In Handbook of Volatility Models and Their Applications, ed. L. Bauwens, C. Hafner and S. Laurent. Hoboken, NJ: John Wiley \& Sons Inc.

Ruiz, E. (1994). Quasi-maximum likelihood estimation of stochastic volatility models. Journal of Econometrics, 63 (1), 289-306

Sandmann, G. and S.J. Koopman (1998). Estimation of stochastic volatility models via Monte Carlo maximum likelihood. Journal of Econometrics, 87 (2), 271-301

Shephard, N. (1996). Statistical Aspects of ARCH and Stochastic Volatility. In Time Series Models in Econometrics, Finance and Other Fields, ed. D.R. Cox, D.V. Hinkley and O.E. Barndorff-Nielsen. London: Chapman and Hall, 1-67.

Shephard, N. and T.G. Andersen (2009). Stochastic Volatility: Origins and Overview. Handbook of Financial Econometrics, Part 2, 233-254.

Shephard, N. and M.K. Pitt (1997). Likelihood Analysis of Non-Gaussian Measurement Time Series. Biometrika, 84, 653-667.

Silvennoinen, A. and T. Teräsvirta, (2009). Multivariate GARCH Models. In Handbook of Financial Time Series, ed. T.G. Andersen, R.A. Davis, J.-P. Kreiss and T. Mikosch. New York: Springer.

Taylor, S.J. (1982). Financial returns modelled by the product of two stochastic processes - a study of daily sugar prices 1961-79. In Time Series Analysis: Theory and Practice, ed. O.D. Anderson, 1, 203-226. Amsterdam: North-Holland.

Taylor, S.J. (1986). Modelling Financial Time Series. Chichester: John Wiley.

Taylor, S.J. (1994). Modelling Stochastic Volatility: A Review and Comparati ve Study. Mathematical Finance, 4 (2), 183-204.

Tsay, R.S. (2005). Analysis of Financial Time Series: Financial Econometrics. (2ed). New York: Wiley.

Tsui, A.K and Q. Yu (1999). Constant conditional correlation in a bivariate garch model: Evidence from the stock market in China. Mathematics and Computers in Simulation, 48, 503-509. 
Watanabe, T. (1999). A Nonlinear Filtering Approach to Stochastic Volatility Models with an Application to Daily Stock Returns. Journal of Applied Econometrics, 14, 101-121

Yu, J. (2002). Forecasting volatility in the New Zealand stock market. Applied Financial Economics, 12, 193-202.

Yu, J. and R. Meyer (2006). Multivariate Stochastic Volatility Models: Bayesian Estimation and Model Comparison. Econometric Reviews, 25, 2-3. 